\title{
RICERCHE SUI FASCI DI QUADRICHE IN UNO SPAZIO $\mathrm{AD} n$ DIMENSIONI.
}

\author{
Memoria di Luigi Brusotti (Sondrio).
}

Adunanza del 10 febbrajo 1907.

Il Prof. Segre, dedicando la seconda parte di una sua classica memoria *) allo studio dei fasci di quadriche in uno spazio ad un numero qualunque di dimensioni, ne ha dimostrate le principali proprietd projettive e ne ha stabilita una classificazione in tipi fondata sul fecondo concetto di divisore elementare già introdotto dal WEIERSTRASs **). Il caso dei fasci di quadriche specializzate, esplicitamente escluso dalla precedente ricerca, è oggetto di un altro lavoro dello stesso Prof. SEGRE in relazione con alcuni sviluppi algebrici del Kronecker ${ }^{* * *}$ ). A queste pubblicazioni si connette una nota del Prof. BerTINI svolta con metodo geometrico $\dagger$ ). Infine il sig. MedicI in un recente lavoro torna incidentalmente sull'argomento, ed una esposizione sistematica di questo si trova nel recentissimo trattato del Prof. Bertini H).

La lettura di una breve Nota del Prof. Gundelfinger sulle quadriche di un fascio conjugate in doppio modo $\left.+t^{+}\right) \mathrm{mi}$ ha indotto a pensare che la teoria potesse trovare ulteriore sviluppo in un confronto metodico fra la geometria del fascio come forma dello spazio ambiente e la geometria del fascio come ente razionale $\infty^{\mathrm{I}}$. In tale indirizzo (gid seguito in modo elementare per il piano e lo spazio ordinario dai migliori trattati) e coi metodi del calcolo simbolico si svolge il presente lavoro.

*) SEGRE, Studio sulle quadriche in uno spazio lineare ad un numero qualunque di dimensioni [Mem. R. Accademia delle Scienze di Torino, serie II, vol. XXXVI (I884), pp. 3-86].

*) WeIErstrass, Zur Theorie der bilinearen und quadratischen Formen [Monatsber. der k. Akademie der Wissenschaften zu Berlin (1868), pag. 310].

***) SEGRE, Ricerche sui fasci di coni quadrici in uno spazio lineare qualunque [Atti R. Accademia delle Scienze di Torino, vol. XIX (1884), pp. 878-897]; KroNecker, Bemerkungen [Monatsber. der k. Akademie der Wiss. zu Berlin (I868), pag. 339]; Ueber Schaaren von quadratischen Formen (Monatsber. (1874), pag. 59].

t) Bertini, Sui fasci di quadriche in uno spazio ad $n$ dimensioni [Rend. R. Accademia dei Lincei, serie IV, vol. II (1886), pp. 208-2 I I].

t†) MedicI, Sulle omografie e correlazioni non singolari in uno spazio ad un numero qualunque di dimensioni [Giorn. di Mat., XLIV (1906), pp. 189-239], Cfr. $\ 9, \mathrm{n}^{\circ} 5 \mathrm{I}$; BERTINI, Introduzione alla geometria projettiva degli iperspazii (Pisa, Enrico Spoerri, 1907), Cfr. Cap. 7.

tff) Arkiv der Math. und Phys. (Leipzig), Bd. III (1902), pp. 75-76. 
Il $\int$ I contiene alcuni sviluppi preliminari (in parte sostanzialmente noti), tra $\mathrm{i}$ quali ricordo il significato geometrico dato al $\mathrm{n}^{0} 3$ per l'annullarsi dell'invariante $\left(a^{\prime} a^{\prime \prime} \ldots a^{(k)} b^{\prime} b^{\prime \prime} \ldots b^{(n-k+1)}\right)^{2}$ di due quadriche $a_{x}^{2}=0, b_{x}^{2}=0$ dello spazio $S_{n}$ ad $n$ dimensioni. Nel $\ 2$, introdotti il fascio $[f]$ e il discriminante $x_{\lambda}^{n+1}$ della sua quadrica $f_{\lambda}$ di parametro $\lambda_{1}: \lambda_{2}$, è assegnato il significato della forma polare $\alpha_{\mu} \alpha_{\nu} \ldots \alpha_{\rho}$ e ne sono dedotte alcune notevoli conseguenze $\left(\mathrm{n}^{\mathrm{i}} 6\right.$ e 9 ); inoltre ( $\mathrm{n}^{\circ}$ I0) vengono definiti e studiati i combinanti binari elementari di $[f]: \alpha_{\lambda}^{n+1}, \beta_{\lambda}^{n}, \ldots \theta_{\lambda}^{k}, \ldots \tau_{\lambda}^{2}, \omega_{\lambda}$, dei quali il primo è il discriminante, mentre quello generico $H_{\lambda}^{k}$ contiene le coordinate iperplanari di spazio $S_{k-1}$ e col suo annullarsi da la condizione perchè l' $S_{k-1}$ tocchi $f_{\lambda}$. Il $\ 3$ tratta del sistema lineare $(\Phi \beta)^{n}=0$ di inviluppi di seconda classe e specialmente della riduzione prodotta sulla sua dimensione $n$ dalla presenza in $[f]$ di quadriche più volte specializzate ( $\mathrm{n}^{\mathrm{i}}$ I I e I 2$)$, come pure di alcuni sistemi ad esso subordinati ( $\mathrm{n}^{\mathrm{i}}$ I 3 e I $_{4}$ ), tra i quali noto una schiera che dico associata al fascio. Il $\ 4$ estende alcuni dei risultati del precedente al sistema di complessi quadratici $(\Pi \theta)^{k}=0$. Al $\ 5$ le principali questioni svolte nel $\ 3$ vengono riprese nell'ipotesi che esista (almeno) una piramide di $n+$ I vertici polare rispetto a tutte le quadriche di $[f]$. Infine il $\ 6$ e dedicato ai fasci pei quali il gruppo dei coni è mutato in sè da un'omografia binaria ciclica d'indice $n+\mathrm{I}$.

In una prossima pubblicazione mi occuperó del caso $n=3$, in particolare.

\section{$\oint$ I. Preliminari.}

I. In uno spazio $S_{n}$ lineare ad $n$ dimensioni siano $x_{1}, x_{2}, \ldots x_{n+1}$ le coordinate projettive omogenee di un punto e sia:

$$
\left(a_{1} x_{x}+a_{2} x_{2}+\cdots+a_{n+1} x_{n+1}\right)^{2}=a_{x}^{2}=a_{x}^{\prime 2}=a_{x}^{\prime \prime 2}=\cdots=0
$$

l'equazione simbolica di una quadrica $f$. Condizione necessaria e sufficiente perchè $f$ abbia un punto doppio [sia specializzata $\left.{ }^{*}\right)$ ] è la coesistenza delle $n+$ I equazioni:

$$
a_{y^{\prime}} a_{x}=0, \quad a_{y^{\prime \prime}} a_{x}=0, \ldots a_{y^{(n+1)}} a_{x}=0,
$$

dove le $y_{i}^{(h)}(b=\mathrm{I}, 2, \ldots n+\mathrm{I})$ sono le coordinate di $n+\mathrm{I}$ punti linearmente indipendenti. Eliminando, alle (1) sostituisco:

$$
\left(a^{\prime} a^{\prime \prime} \ldots a^{(n+\mathrm{I})}\right) a_{y^{\prime}}^{\prime} a_{y^{\prime \prime}}^{\prime \prime} \ldots a_{y^{(n+1)}}^{(n+\mathrm{I})}=0
$$

che, colla permutazione dei simboli, prende la forma

$$
\left(a^{\prime} a^{\prime \prime} \ldots a^{(n+\mathrm{I})}\right)^{2}\left(y^{\prime} y^{\prime \prime} \ldots y^{(n+\mathrm{s})}\right)=0 .
$$

Il secondo fattore, per l'indipendenza degli $n+\mathrm{I}$ punti, è $\neq 0$, onde la (3) è equivalente $a$ :

(4)

$$
\left(a^{\prime} a^{\prime \prime} \ldots a^{(n+\mathrm{s})}\right)^{2}=0 .
$$

Cioè: L'annullarsi di $\left(a^{\prime} a^{\prime \prime} \ldots a^{(n+1)}\right)^{2}$ (discriminante) è condizione necessaria e sufficiente perchè $f$ sia specializzata.

") Denominazione di SzGre, Studio sulle quadriche, etc., Parte I; $\ \mathrm{I} ; \mathrm{n}^{\circ}$ is e seg. 
Un $S_{p}$ (di $S_{n}$ ) sia individuato da $p+\mathrm{I}$ punti di coordinate $y_{i}^{(h)}(h=\mathrm{I}, 2, \ldots p+\mathrm{I})$ o come intersezione di $n-p$ iperpiani di coordinate $u_{i}^{(k)}(k=1,2, \ldots n-p)$. E sempre possibile assegnare $n-p$ punti, linearmente indipendenti insieme coi $p+\mathrm{I}$ gia presi, di coordinate $z_{i}^{(k)}(k=\mathrm{I}, 2, \ldots n-p)$ in modo che il punto di coordinate $z_{i}^{(k)}$ giaccia su tutti gli iperpiani scelti escluso quello di coordinate $u_{i}^{(k)}$. Si ha cosi:

$$
\left\{\begin{array}{c}
u_{y^{(b)}}^{(k)}=0 \\
u_{z^{(k)}}^{(k)}=0 \\
u_{z^{(k)}}^{(k)} \neq 0
\end{array}\right.
$$

Condizione necessaria e sufficiente perchè $S_{p}$ tocchi $f$ è la coesistenza delle:

$a_{y^{\prime}} a_{x}=0, \quad a_{y^{\prime \prime}} a_{x}=0, \ldots a_{y^{(p+1)}} a_{x}=0 ; \quad u_{x}^{\prime}=0, u_{x}^{\prime \prime}=0, \ldots u_{x}^{(n-p)}=0$, alle quali, eliminando, sostituisco:

$$
\left(a^{\prime} a^{\prime \prime} \ldots a^{(p+1)} u^{\prime} u^{\prime \prime} \ldots u^{(n-p)}\right) a_{y^{\prime}}^{\prime} a_{y^{\prime \prime}}^{\prime \prime} \ldots a_{y^{(p+1)}}^{(p+1)}=0
$$

od anche [cfr. le $(s)]$ :

$$
\left(a^{\prime} a^{\prime \prime} \ldots a^{\left(p+1^{\prime}\right.} u^{\prime} u^{\prime \prime} \ldots u^{(n-p)}\right) a_{y^{\prime}}^{\prime} a_{y^{\prime \prime}}^{\prime \prime} \ldots a_{y^{(p+\mathrm{I})}}^{(p+\mathrm{r})} u_{x^{\prime}}^{\prime} u_{z^{\prime \prime}}^{\prime \prime} \ldots u_{z^{(n-p)}}^{(n-p)}=0 .
$$

In (6) posso permutare in tutti i modi possibili i simboli equivalenti $a^{\prime} a^{\prime \prime} \ldots a^{(p+x)}$ e sommare; ma posso anche più in generale operare in tale maniera su tutte le lettere $a$, $u$, essendo nulli, per le $(\zeta)$, i termini provenienti da permutazioni che producano scambio di due lettere $u$ o di una lettera $a$ con una lettera $u$. Alla (6) sostituisco quindi :

$$
\left(a^{\prime} a^{\prime \prime} \ldots a^{(p+1)} u^{\prime} u^{\prime \prime} \ldots u^{(n-p)}\right)^{2}\left(y^{\prime} y^{\prime \prime} \ldots y^{(p+1)} z^{\prime} z^{\prime \prime} \ldots z^{(n-p)}\right)=0
$$

e, per l'indipendenza dei punti considerati, più semplicemente:

\section{Segue :}

$$
\left(a^{\prime} a^{\prime \prime} \ldots a^{(p+1)} u^{\prime} u^{\prime \prime} \ldots u^{(n-p)}\right)^{2}=0 .
$$

L'annullarsi $d i\left(a^{\prime} a^{\prime \prime} \ldots a^{(p+1)} u^{\prime} u^{\prime \prime} \ldots u^{(n-p)}\right)^{2} \dot{e}$ condizione necessaria e sufficiente perchè l'S intersezione degli iperpiani di coordinate $u_{i}^{(k)}(k=1,2, \ldots n-p)$ tocchi $f$. Il suo annullarsi identico (rispetto alle $u$ ) $\dot{e}$ condizione necessaria e sufficiente perchè $f$ contenga un $S_{n-p}$ di punti doppî (sia $n-p+\mathrm{I}$ volte specializzata).

$\mathrm{La}$ (7) si puó considerare come un'equazione nelle coordinate iperplanari di un $S_{p}$ e quindi, quando non sia identica, rappresenta il complesso (quadratico) degli $S_{p}$ tangenti ad $f$.

2. Date due quadriche $f$ e $g$, rispettivamente di equazioni:

$$
\begin{aligned}
& a_{x}^{2}=a_{x}^{\prime 2}=a_{x}^{\prime \prime 2}=\cdots=0 \\
& b_{x}^{2}=b_{x}^{\prime 2}=b_{x}^{\prime \prime 2}=\cdots=0
\end{aligned}
$$

si consideri il loro invariante simultaneo :

$$
[k, n-k+\mathrm{I}]=\left(a^{\prime} a^{\prime \prime} \ldots a^{(k)} b^{\prime} b^{\prime \prime} \ldots b^{(n-k+\mathrm{I})}\right)^{2}
$$

per $k=0,1,2, \ldots n, n+\mathrm{I}$.

Per $k=\mathrm{o}(k=n+\mathrm{I})$ esso (cfr. $\left.\mathrm{n}^{\circ} \mathrm{I}\right)$ coincide col discriminante di $g($ di $f)$. 
Per $k=\mathbf{I}(k=n)$, come ha dimostrato Rosanes $\left.{ }^{*}\right)$, l'annullarsi di tale invariante è condizione necessaria e sufficiente per l'esistenza di una piramide di $n+1$ vertici, inscritta in $f$ (in $g$ ) e polare rispetto a $g$ (ad $f$ ); o anche per l'esistenza di una piramide di $n+$ I vertici circoscritta a $g$ (ad $f$ ) e polare rispetto ad $f($ a $g$ ). Lo stesso autore ha notato [1. c.] che, se esiste una di tali piramidi ne esistono $\infty^{\frac{n(n-1)}{2}}$. Il Prof. SEGRE, prendendo occasione dal lavoro citato, ha dimostrato che l'annuilarsi di $[k, n-k+\mathrm{I}]$ è condizione necessaria per l'esistenza di due piramidi di $n+$ I vertici polari reciproche rispetto a $g$ e di cui gli $S_{k-1}$ corrispondenti siano conjugati rispetto ad $f$; ha osservato che la condizione è pure sufficiente quando sia

$$
n(n+\mathrm{I}) \supseteq\left(\begin{array}{c}
n+\mathrm{I} \\
k
\end{array}\right)-\mathrm{I}
$$

ed ha posto il problema, tuttora insoluto, della sufficienza di tale condizione in generale ${ }^{* *}$ ).

Nel numero seguente, abbandonando la via tracciata dai lavori qui ricordati, stabilisco un significato dell'annullarsi di $[k, n-k+\mathrm{I}]$, che si fonda sopra un altro concetto.

3. E noto che gli $S_{k-1}$ secanti un dato $S_{n-k}$ formano un complesso lineare (speciale); il complesso quadratico che si ottiene contando due volte tale complesso lineare sard̀ detto per brevità complesso quadratico degenere avente per sostegno il dato $S_{n-k}$. Cio posto:

L'annullarsi di $[k, n-k+\mathrm{I}]$ è condizione necessaria e sufficiente perchè il complesso degli $S_{k-1}$ tangenti ad $f$ giaccia nel sistema lineare determinato dai complessi quadratici degeneri aventi per sostegni gli $S_{n-k}$ tangenti a $g$.

x) La condizione è necessaria. Se infatti il complesso:

$$
\left(a^{\prime} a^{\prime \prime} . \ldots a^{(k)} u^{\prime} u^{\prime \prime} \ldots u^{(n-k+1)}\right)^{2}=0
$$

giace in tale sistema lineare, si ha identicamente

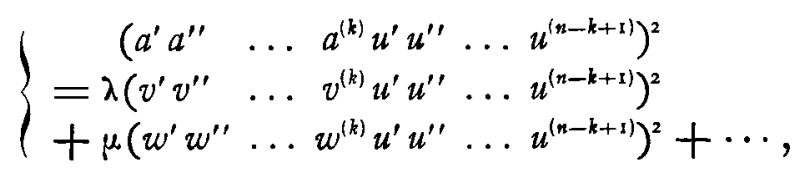

*) Erweiterung eines bekannten Satzes auf Formen von beliebig vielen Veränderlichen [Math. Ann., vol. XXIII (1884), pp. 4I2-4I5]. Ometto la citazione dei numerosi e noti lavori che si riferiscono ai casi $n=2, n=3$.

**) SEGRE, Sur les invariants stmultanés de deux formes quadratiques; estratto di lettera a RosanES [Math. Ann., vol. XXIV (1884), pp. I 52-156]. Dal teorema di SEGRE si deduce come corollario che in $S_{3}$ l'annullarsi di $[2,2]$ è condizione necessaria e sufficiente per l'esistenza di un tetraedro polare rispetto ad una delle quadriche e cogli spigoli tangenti all'altra. Per questa proposizione vedi: Loroth, Ueber Polartetraeder und die Schnittcurve zweier Flächen zweiter Ordnung [Zeitschrift f. Math. und Phys., Leipzig, vol. XIII (1868), pag. 404]; Voss, Die Liniengeometrie in ibrer Anwendung auf die Flächen zweiten Grades [Math. Ann., vol. X (1876), pp. 143-188]; VoGT, Sur les tétraèdres conjugués par rapport à une quadrique et dont les arêtes sont tangentes à une autre qu..drique [Ann. de l'Éc. norm., III érie, t. XII (I 895 ), pp. 363-389]. 
dove l' $S_{n-k}$ intersezione degli iperpiani di coordinate $\tau_{1}^{(b)}(b=\mathrm{I}, 2, \ldots k), 1 S_{n-k}$ intersezione di quelli di coordinate $w_{i}^{(b)}(b=\mathrm{I}, 2, \ldots k)$, ecc. sono tangenti a $g$, onde si ha:

$$
\begin{aligned}
& \left(v^{\prime} v^{\prime \prime} \ldots v^{(k)} b^{\prime} b^{\prime \prime} \ldots b^{(n-k+1)}\right)^{2}=0 \\
& \left(w^{\prime} w^{\prime \prime} \ldots w^{(k)} b^{\prime} b^{\prime \prime} \ldots b^{(n-k+1)}\right)^{2}=0
\end{aligned}
$$

Segue che se in (8) alle serie di variabili contragredienti $u^{\prime}, u^{\prime \prime}, \ldots u^{(n-k+1)}$ si sostituiscono le serie di simboli $b^{\prime}, b^{\prime \prime}, \ldots b^{(n-k+1)}$ il secondo membro si annulla, ed è quindi, come si voleva:

$$
\left(a^{\prime} a^{\prime \prime} \ldots a^{(k)} b^{\prime} b^{\prime \prime} \ldots b^{(n-k+1)}\right)^{2}=0 .
$$

(3) La condizione è sufficiente. Sia infatti:

e siano:

$$
\left(a^{\prime} a^{\prime \prime} \ldots a^{(k)} b^{\prime} b^{\prime \prime} \ldots b^{(n-k+\mathrm{I})}\right)^{2}=0
$$

(9)

$$
\Phi_{1}=0, \quad \Phi_{2}=0, \ldots
$$

relazioni lineari nei coefficienti dell'equazione generale di un complesso quadratico di spazii $S_{k-1}$, le quali bastino ed occorrano per determinare il sistema lineare sopra indicato. Se nelle (9) pongo i coefficienti dell'equazione del complesso degenere:

$$
\left(v^{\prime} v^{\prime \prime} \ldots v^{(k)} u^{\prime} u^{\prime \prime} \ldots u^{(n-k+\mathrm{I})}\right)^{2}=\mathrm{o},
$$

esse divengono relazioni quadratiche nelle coordinate dell' $S_{n-k}$ sostegno, relazioni necessariamente soddisfatte da quelle di ogni $S_{n-k}$ tangente a $g$, per la definizione geometrica del sistema lineare. Ma gli $S_{n-k}$ tangenti a $g$ soddisfano alla sola condizione di giacere nel complesso quadratico $\left(u^{\prime} u^{\prime \prime} \ldots u^{(k)} b^{\prime} b^{\prime \prime} \ldots b^{(n-k+\mathrm{I})}\right)^{2}=\mathrm{o}$ (anzi non sono sottoposti ad alcuna se $g$ è almeno $k+$ I volte specializzata), onde si ha che, per la sostituzione indicata, $\Phi_{m}=0$ si muta in:

$$
\rho_{m}\left(v^{\prime} v^{\prime \prime} \ldots v^{(k)} b^{\prime} b^{\prime \prime} \ldots b^{(n-k+1)}\right)^{2}+\varphi_{m}\left[v^{\prime} v^{\prime \prime} \ldots v^{(k)}\right]=0,
$$

dove $\rho_{m}$ è una costante e $\varphi_{m}\left[v^{\prime} v^{\prime \prime} \ldots v^{(k)}\right]$ come funzione delle coordinate di $S_{n-k}$ si annulla in forza delle relazioni identiche esistenti fra esse, mentre come funzione delle $v_{i}^{(h)} \dot{e}$ identicamente zero ed è solo stata scritta per mantenere alla (II) il suo aspetto formale. Pongo ora nelle (9) i coefficienti dell'equazione del complesso:

$$
\left(a^{\prime} a^{\prime \prime} \ldots a^{(k)} u^{\prime} u^{\prime \prime} \ldots u^{(n-k+1)}\right)^{2}=0 .
$$

Per questo, poichè (I2) si deduce da (Io) sostituendo alle variabili $v^{(h)}$ i simboli $a^{(h)}$, basta porre in (II) invece delle $v^{(b)}$ le $a^{(b)}$, onde $\Phi_{m}=0$ si trasforma in:

$$
p_{m}\left(a^{\prime} a^{\prime \prime} \ldots a^{(k)} b^{\prime} b^{\prime \prime} \ldots b^{(n-k+\mathrm{r})}\right)^{2}+\varphi_{m}\left[a^{\prime} a^{\prime \prime} \ldots a^{(k)}\right]=0 .
$$

Il primo termine di (I3) è nullo per l'ipotesi, ed il secondo è nullo perchè ottenuto sostituendo i simboli alle variabili nell'espressione $\varphi_{m}\left[v^{\prime} v^{\prime \prime} \ldots v^{(k)}\right]$ identicamente nulla. Si deduce quindi, cone si voleva, che i coefficienti di (12) soddisfano alle (9) ${ }^{*}$ ).

*) Dal teorema ora dimostrato pare si possa dedurre che l'invariante $[k, n-k+1]$ trova specialmente il suo posto nella geometria degli $S_{k-1}$ (e dei loro duali $S_{n-k}$ ) in $S_{n}$, osservazione questa che per l'invariante $[2,2]$ di due quadriche in $S_{3}$ è già contenuta nel citato lavoro di Voss (v. pag. I 70). 
4. Nel seguito, dovendo considcrare due quadriche $f, g$, per le quali si annulla l'invariante $[k, n-k+\mathrm{I}]$, dirò che $f$ ì $k$-armonica rispetto a $g$ (e che $g$ è $n-k+\mathrm{I}$-armonica rispetto ad $f)$. Se in particolare si annulla $[\mathrm{I}, n]$, dirò che $f$ e $g$ sono cunjugate, considerate rispettivamente come luogo e come inviluppo.

Cio posto, stabilisco qui due semplici osservazioni in modo diretto, per quanto siano strettamente legate al teorema del $n^{\circ} 3$; e cioè:

a) Una quadrica $n-k+2$ volte specializzata è $k$-armonica rispetto ad ogni altra quadrica; e reciprocamente. Se infatti $f$ è $n-k+2$ volte specializzata, è identicamente:

$$
\left(a^{\prime} a^{\prime \prime} \ldots a^{(k)} u^{\prime} u^{\prime \prime} \ldots u^{(n-k+1)}\right)^{2}=0,
$$

onde anche, qualunque sia $b_{x}^{2}$ :

$$
\left(a^{\prime} a^{\prime \prime} \ldots a^{(k)} b^{\prime} b^{\prime \prime} \ldots b^{(n-k+1)}\right)^{2}=0 .
$$

Reciprocamente, qualunque sia $b_{x}^{2}$, sussista la

$$
\left(a^{\prime} a^{\prime \prime} \ldots a^{(k)} b^{\prime} b^{\prime \prime} \ldots b^{(n-k+\mathrm{I})}\right)^{2}=0
$$

che si deduce dalla

$$
\left(u^{\prime} u^{\prime \prime} \ldots u^{(k)} b^{\prime} b^{\prime \prime} \ldots b^{(n-k+1)}\right)^{2}=0
$$

sostituendo le $a^{(b)}$ alle $u^{(h)}$. Se come $b_{x}^{2}=$ o si prende una quadrica $k$ volte specializzata il cui $S_{k-1}$ doppio è intersezione degli iperpiani di coordinate $v_{i}^{(b)}(b=\mathrm{I}, 2, \ldots n-k+\mathrm{I})$ e si ricorda che gli $S_{n-k}$ tangenti ad una quadrica $k$ volte specializzata sono quelli e solo quelli che tagliano l' $S_{k-1}$ doppio, nel caso particolare (IS) si puó scrivere nel seguente modo:

$$
\left(u^{\prime} u^{\prime \prime} \ldots u^{(k)} v^{\prime} v^{\prime \prime} \ldots v^{(n-k+1)}\right)^{2}=0
$$

e quindi (I4) nel seguente:

$$
\left(a^{\prime} a^{\prime \prime} \ldots a^{(k)} v^{\prime} v^{\prime \prime} \ldots v^{(n-k+1)}\right)^{2}=0
$$

il che, per l'arbitrarietà delle $v_{i}^{(h)}$, dimostra quanto si voleva.

.) Una quadrica $n-k+\mathrm{I}$ volte specializzata è $k$-armonica rispetto alle quadriche tangenti l' $S_{n-k}$ doppio e solo ad esse.

Per un'osservazione precedente l'equazione del complesso degli $S_{k-1}$ tangenti alla quadrica $a_{x}^{2}=0, n-k+I$ volte specializzata, puó prendere le due forme:

$$
\begin{aligned}
& \left(a^{\prime} a^{\prime \prime} \ldots a^{(k)} u^{\prime} u^{\prime \prime} \ldots u^{(n-k+1)}\right)^{2}=0 \\
& \left(v^{\prime} v^{\prime \prime} \ldots v^{(k)} u^{\prime} u^{\prime \prime} \ldots u^{(n-k+1)}\right)^{2}=0
\end{aligned}
$$

quando si supponga l'S $S_{n-k}$ doppio intersezione degli iperpiani di coordinate $v_{i}^{(b)}(b=\mathrm{I}, 2, \ldots k)$.

Qualche lettore potrà anche trovare una certa analogia fra il presente teorema e quello ben noto di Rosanes sulle forme binarie conjugate $[\mathrm{v}$. Ueber die Darstellung binärer Formen als Potenzsummen; Journal für die reine und angewandte Mathematik, vol. LXXV (1873). pp. I72-176], la cui estensione può dare una semplice interpretazione dell'annullarsi dell'invariante bilineare di una ipersuperficie (di ordine $m$ ) e di un inviluppo (di classe $m$ ) in $S_{n}$, come per $S_{3}$ ha stabilito REYe in un suo classico lavoro [v. Ueber algebraische Flächen, die zu einander apolar sind; Journal für die reine und angewandte Mathematik, vol. LXXIX (I875), pp. I59-175; e specialmente a pag. I65]. 
Segue che la condizione:

può pure scriversi :

$$
\left(a^{\prime} a^{\prime \prime} \ldots a^{(k)} b^{\prime} b^{\prime \prime} \ldots b^{(n-k+1)}\right)^{2}=0
$$

$$
\left(v^{\prime} v^{\prime \prime} \ldots v^{(k)} b^{\prime} b^{\prime \prime} \ldots b^{(n-k+1)}\right)^{2}=0
$$

il che dimostra il teorema.

5. Date $k$ quadriche $c_{x}^{2}=0, d_{x}^{2}=0, \ldots, s_{x}^{2}=0$, resta in generale determinato il complesso quadratico di spazii $S_{k-1}$ di equazione:

$$
\left(c d \ldots s u^{\prime} u^{\prime \prime} \ldots u^{(n-k+1)}\right)^{2}=0 .
$$

Poichè più innanzi si incontreranno complessi di questo tipo, non è inopportuno osservare come il teorema del $\mathrm{n}^{0} 3$ sia in proposito suscettibile di una generalizzazione. Infatti nella dimostrazione di quel teorema non si fa uso dell'equivalenza dei simboli $a^{\prime} a^{\prime \prime} \ldots a^{(k)}$, onde essa rimane valida anche quando a questi si sostituiscano quelli non equivalenti $c, d, \ldots s$. Si giunge cosi al risultato :

$L^{\prime} a n n u l l a r s i d i\left(c d \ldots s b^{\prime} b^{\prime \prime} \ldots b^{(n-k+1)}\right)^{2} \dot{e}$ condizione necessaria e sufficiente perchè il complesso $\left(c d \ldots s u^{\prime} u^{\prime \prime} \ldots u^{(n-k+1)}\right)^{2}=$ o giaccia nel sistema lineare determinato dai complessi quadratici degeneri aventi per sostegni gli $S_{n-k}$ tangenti $a b_{x}^{2}=0$.

Quando si annulli $\left(c d \ldots s b^{\prime} b^{\prime \prime} \ldots b^{(n-k+1)}\right)^{2}$, diró che il complesso $\left(c d \ldots s u^{\prime} u^{\prime \prime} \ldots u^{(n-k+1)}\right)^{\prime}=0$ e la quadrica $b_{x}^{2}=0$ sono armonici.

Cio posto, le osservazioni $\alpha$ ), $\beta$ ) del $\mathrm{n}^{\circ} 4$ si estendono come segue :

$\left.\alpha^{\prime}\right)$ Se una quadrica $\dot{e} n-k+2$ volte specializáata, $\dot{e}$ armonica ad ogni complesso di tipo $\left(u^{\prime} u^{\prime \prime} \ldots u^{(k)} c d \ldots r\right)^{2}=$ o e reciprocamente.

$\left.\beta^{\prime}\right)$ Una quadrica $n-k+\mathrm{I}$ volte specializzata $\dot{e}$ armonica ai complessi di tiso $\left(u^{\prime} u^{\prime \prime} \ldots u^{(i)} c d \ldots r\right)^{2}=$ o contenenti l'S $S_{r, k}$ doppio e solo ad essi.

Chiudo questi preliminari osservando che nel seguito verrd pure considerato l'invariante :

$$
(c d \ldots t)^{2}
$$

di $n+$ I quadriche $c_{x}^{2}=0, d_{x}^{2}=0, \ldots t_{x}^{2}=0$, il quale sarà detto il loro invariante $n+\mathrm{I}$-lineare.

\section{$\int$ 2. Il fascio di quadriche ed $i$ suoi combinanti binarî.}

6. Due quadriche distinte :

$$
b_{x}^{2}=0, \quad c_{x}^{2}=0
$$

determinano un fascio [f], la cui quadrica generica $f_{\lambda}$ è di equazione

$$
\lambda_{1} b_{x}^{2}+\lambda_{2} c_{x}^{2}=0
$$

La (I) sarà simbolicamente rappresentata con:

$$
\left(a_{\lambda_{1}} x_{1}+a_{\lambda_{2}} x_{2}+\cdots+a_{\lambda, n+1} x_{n+1}\right)^{2}=a_{\lambda x}^{2}=a_{\lambda x}^{\prime 2}=a_{\lambda_{x}}^{\prime \prime 2}=\cdots=0 .
$$

Ogni formazione invariantiva di $a_{\lambda x}^{2}$, contenente eventualmente anche coordinate di spazii, è in generale una forma binaria in $(\lambda)$ e da questo punto di vista sarà detta 
un combinante binario del fascio. Allo studio dei più semplici fra i combinanti premetto un teorema generale, che avrd in seguito più di una applicazione.

Se si pone:

$$
\Psi_{\lambda}^{k}=\left(a_{\lambda}^{\prime} a_{\lambda}^{\prime \prime} \ldots a_{\lambda}^{(k)} d e \ldots r\right)^{2} \text {, }
$$

dove le $d, e, \ldots r$ sono simboli (o variabili contragredienti), ’̀ pure:

Infatti si ha :

$$
\Psi_{\lambda}^{k-1} \Psi_{\mu}=\left(a_{\lambda}^{\prime} a_{\lambda}^{\prime \prime} \ldots a_{\lambda}^{(k-1)} a_{\mu} d e \ldots r\right)^{2} .
$$

$$
\Psi_{\lambda}^{k}=\left(\sum \pm a_{\lambda_{1}}^{\prime} a_{\lambda h_{2}}^{\prime \prime} \ldots a_{\lambda b_{k}}^{(k)} d_{b_{k+1}} e_{h_{k+2}} \ldots r_{h_{n+1}}\right)^{2}
$$

dove $b_{1}, h_{2}, \ldots b_{n+1}$ è una permutazione (da prendersi in tutti i modi) di I, $2, \ldots n+\mathrm{I}$ ed il segno è t o - secondo che la permutazione è di classe pari o dispari. È dunque anche:

$$
\Psi_{\lambda}^{k}=\sum \pm a_{\lambda_{i_{1}}}^{\prime} a_{\lambda_{j_{1}}}^{\prime} a_{\lambda_{i_{2}}}^{\prime \prime} a_{\lambda_{j_{2}}}^{\prime \prime} \ldots a_{\lambda_{i_{k}}}^{(k)} a_{\lambda_{j_{k}}}^{(k)} d_{i_{k+1}} d_{j_{k+1}} \ldots r_{i_{n+1}} r_{j_{n+1}}
$$

dove $i_{1}, i_{2}, \ldots i_{n+1} ; j_{1}, j_{2}, \ldots j_{n+1}$ sono permutazioni di I, $2, \ldots n+$ r, ciascuna da prendersi in tutti $\mathrm{i}$ modi possibili ed il segno è $+\mathrm{o}-$ secondo che le due permutazioni sono di uguale o di diversa classe. Poichè ciascuno dei prodotti simbolici $a_{\lambda_{i_{m}}} a_{\lambda_{j_{m}}}=\lambda_{1} b_{i_{m}} b_{j_{m}}+\lambda_{2} c_{i_{m}} c_{j_{m}}$ è lineare in $(\lambda)$, si deduce :

$$
\begin{aligned}
& \Psi_{\lambda}^{k-1} \Psi_{\mu}=\frac{I}{k}\left[\sum \pm a_{\mu i_{1}} a_{\mu j_{1}} a_{\lambda_{i_{2}}}^{\prime \prime} a_{\lambda_{j_{2}}}^{\prime \prime} \ldots a_{\lambda_{i_{k}}}^{(k)} a_{\lambda_{j_{k}}}^{(k)} d_{i_{k+1}} d_{j_{k+1}} \ldots r_{i_{n+1}} r_{j_{n+1}}\right. \\
& +\sum \pm a_{\lambda_{1}}^{\prime} a_{\lambda_{j_{1}}}^{\prime} a_{\mu i_{2}} a_{\mu j_{2}} \ldots a_{\lambda_{i_{k}}}^{(k)} a_{\lambda_{j_{k}}}^{(k)} d_{i_{k+1}} d_{j_{k+1}} \ldots r_{i_{n+1}} r_{j_{n+1}} \\
& +\ldots \ldots \ldots \ldots \ldots \ldots \\
& \left.+\sum \pm a_{\lambda_{i_{1}}}^{\prime} a_{\lambda_{j_{1}}}^{\prime} a_{\lambda_{i_{2}}}^{\prime \prime} a_{\lambda_{j_{2}}}^{\prime \prime} \ldots a_{\mu_{i_{k}}} a_{\mu j_{k}} d_{i_{k+1}} d_{j_{k+1}} \ldots r_{i_{k+1}} r_{j_{n+1}}\right] \text {. }
\end{aligned}
$$

Ciascuno dei sommatorî del secondo membro, tenuta presente l'equivalenza dei simboli $a_{\lambda}$, vale $\left(a_{\lambda}^{\prime} a_{\lambda}^{\prime \prime} \ldots a_{\lambda}^{(k-1)} a_{\mu} d e \ldots r\right)^{2}$; onde è, come si voleva :

$$
\Psi_{\lambda}^{k-1} \Psi_{\mu}=\left(a_{\lambda}^{\prime} a_{\lambda}^{\prime \prime} \ldots a_{\lambda}^{(k-1)} a_{\mu} d e \ldots r\right)^{2} .
$$

7. Fra i combinanti del fascio ha particolare importanza il discriminante:

$$
\alpha_{\lambda}^{n+1}=\alpha_{\lambda}^{\prime n+1}=\cdots=\left(a_{\lambda}^{\prime} a_{\lambda}^{\prime \prime} \ldots a_{\lambda}^{(n+1)}\right)^{2}
$$

che col suo annullarsi dd la condizione perchè $f_{\lambda}$ si specializzi. In generale $\alpha_{2}^{n+1}=0$ rappresenta il gruppo delle $n+$ I quadriche specializzate (coni), non sempre distinte, del fascio [f]; l'identico annullarsi del discriminante, $\alpha_{\lambda}^{n+1} \equiv 0$, è condizione necessaria e sufficiente perchè il fascio sia di quadriche specializzate. Dal teorema del $n^{\circ} 6$, applicato successivamente $n$ volte, si deduce :

$$
x_{\mu} \alpha_{\nu} \ldots \alpha_{p}=\left(a_{\mu} a_{\nu} \ldots a_{p}\right)^{2} .
$$

Se è $\alpha_{\lambda}^{n+1} \equiv 0$, è pure $\alpha_{\mu} \alpha_{y} \ldots \alpha_{\rho} \equiv 0$, onde si ha :

L'invariante $n+\mathrm{I}$ lineare di $n+\mathrm{I}$ quadriche appartenenti ad un fascio di quadriche tutte specializzate è nullo.

In generale invece :

Condizione necessaria e sufficiente perche $n+$ I quadriche di un fascio siano ad invariante $n+\mathrm{I}$-lineare nullo è che nel campo binario del fascio il gruppo delle $n+\mathrm{I}$ 
quadricbe sia coniugato a quello degli $n+\mathrm{I}$ coni. Tali gruppi di quadriche formano dunque nel fascio una involuzione $g_{n+1}^{n}$.

Se si pone:

è pure :

$$
\delta_{\lambda}^{n+1}=(\mu \cdot \lambda)(\nu \lambda) \ldots(p \lambda)
$$

$$
(\alpha \delta)^{n+1}=\alpha_{\mu} \alpha_{\nu} \ldots \alpha_{p}
$$

onde il precedente teorema fornisce il significato dell'annullarsi della spinta $n+\mathrm{I}^{\text {esima }}$ $d i \alpha_{\lambda}^{n+1}$ su di una binaria $\delta_{\lambda}^{n+1}$.

In particolare, quando si escluda il caso $\alpha_{\lambda}^{n+1} \equiv 0$, si ha:

L'annullarsi di $\left(x_{\alpha^{\prime}}\right)^{n+1}$ è condizione necessaria e sufficiente perchè il gruppo dei coni sia ad invariante $n+$ I lineare nullo.

Quindi: L'invariante $n+$ I lineare dei coni di un fascio, per $n$ pari, è sempre nullo.

Come caso particolare della (.2) si deduce:

$$
\alpha_{\mu}^{k} \alpha_{\lambda}^{n-k+1}=\left(a_{\mu}^{\prime} a_{\mu}^{\prime \prime} \ldots a_{\mu}^{(k)} a_{\lambda}^{\prime} a_{\lambda}^{\prime \prime} \ldots a_{\lambda}^{(n-k+1)}\right)^{2} ;
$$

onde: ogni quadrica $f_{\mu}$, in generale, è k-armonica ad $n-k+\mathrm{I}$ quadriche di $[f]$, che nel campo binario del fascio costituiscono il gruppo $k$-esimo potare di $f_{\mu}$ rispeto a quello dei coni. Per $k=\mathrm{I}$ : ogni quadrica $f_{\mu}$, in generale, è coniugate comé lúogo ad $n$ quadriche di $[f]$, costituenti il primo gruppo polare di $f_{\mu}$ rispetto al gruppo dei coni. Per $k=n$ : ogni quadrica $f_{\mu}$, in generale, è coniugata come inviluppo ad una sola quadrica di $[f]$, quella costituente il gruppo n-esimo polare di $f_{\mu}$ rispetto al gruppo dei coni.

Il caso $\alpha_{\lambda}^{n+1} \equiv$ o conduce però al seguente enunciato: In un fascio di quadriche tutte specializate ogni quadrica è k-armonica alle rimanenti.

Se ne deduce, per l'Oss. $\beta$ ) del $\mathrm{n}^{\circ} 4$, che in tal fascio lo spazio doppio di una delle quadriche tocea le rimanenti, ed in particolare il vertice di un cono ordinario giace nella quartica base (quindi ne è punto doppio) *).

Cosi il caso $\alpha_{,}^{n+1} \neq 0, \alpha_{\mu}^{k} \alpha_{\lambda}^{n-k+1} \equiv o$ [ident. rispetto ( $\lambda$ )] conduce al teorema: Condizione necessaria e sufficiente perchè una quadrica di un fascio [f] sia k-armonica a tutte le rimanenti $d i[f]$ è che essa conti $n-k+2$ volte nel grappo dei coni. Tenuta presente l'Oss. $\alpha$ ) di 4 , si ha pure: Una quadrioa $n-k+2$ volte specializzata di [f] conta almeno $n-k+2$ volte nel gruppo dei coni ${ }^{* *}$ ). Tenuta presente invece la già citata (3) di 4, si ha: Se una quadrica $n-k+$ I volte specializzata conta $n-k+2$ volte nol gruppo dei coni, l'S $S_{n-k}$ doppio di essa tocca ogni quadrica del fascio; e reciprocamente. In particolare: Se un cono ordinario conto due volte nel gruppo dei cow, it suo vertice giace sulla quartica base (ne è un punto doppio); e reciprocamente.

Se il discriminante della forma binaria $\alpha_{i}^{n+1}$ è nullo, o la quadrica corrispondente alla radice doppia è un cono ordinario ed allora, pert cio che precede, la quartica base

*) Per l'ultima affermazione cfr.: SEGRE, Ricerche sui fasci di coni quadrici, etc. (cit.), $\mathrm{n}^{\circ}$ s, $\mathrm{n}^{\circ}$ Io; BERTINI, Sui fasci di quadriche, etc. (cit.), $\mathrm{n}^{\mathrm{o}} 4$.

**) Il che è pure conseguenza delle considerazion svolte in: SEGRE, Stidio sulle quadrictje, etc. (cit.), Parte $2^{\mathrm{a}}, \mathbb{3} ; \mathrm{n}^{\circ} 63$. 
possiede un punto doppio nel vertice; o tale quadrica è più volte specializzata ed allora la quartica base possiede $i$ punti doppi risultanti dall'intersezione dello spazio doppio con una quadrica generica di $[f]$. Reciprocamente se la quartica base possiede un punto doppio, si verifica una delle precedenti condizioni geometriche e quindi il discriminante di $\alpha_{\lambda}^{n+1}$ è nullo. Dunque: Condizione necessaria e sufficiente perchè la quartica base di $[f]$ possegga un punto doppio è che si annulli il discriminante di $\alpha_{\lambda}^{n+1}{ }^{*}$ ).

8. Nel presente numero svolgo alcune considerazioni, le quali servono in molti casi a stabilire il significato di forme del campo binario nella geometria di $S_{n}$. Siano due forme $\varphi_{\lambda}^{b}, \psi_{\lambda}^{b}(b \leq n)$, ciascuna delle quali rappresenta col suo annullarsi un gruppo di $b$ quadriche di $[f]$. Uno qualunque degli $\infty^{h(n-b)}$ spazii $S_{b-1}$ tangenti a tutte le quadriche del gruppo $p_{\lambda}^{h}=0$ (per $h=n$ ne esistono $\left.2^{n}\right)$ seca $[f]$ in un fascio $\left[f^{\prime}\right]$, pel quale si puó assumere come discriminante $\varphi_{i}^{b}$, quando si convenga di attribuire il pa-

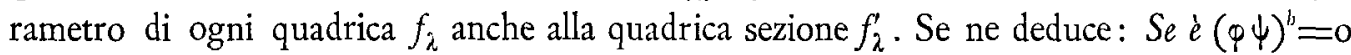
(cioe $i$ gruppi $\psi_{\lambda}^{b}=0, \psi_{\lambda}^{b}=0$ sono coniugati) ogni $S_{b-1}$ tangente a tutte le quadriche di $\varphi_{\lambda}^{h}=0$ [risp. di $\psi_{\lambda}^{h}=0$ ] seca le quadriche di $\psi_{\lambda}^{h}=0$ [risp. di $\varphi_{\lambda}^{h}=0$ ] in $h$ quadriche ad invariante b-lineare nullo. Reciprocamente: Se uno degli $S_{i_{-1}}$ tangenti a tutte le quadriche di $\psi_{\lambda}^{h}=0$ [risp. di $\psi_{\lambda}^{h}=0$ ] seca le quadriche $\psi_{\lambda}^{h}=0$ [risp. $\psi_{\lambda}^{h}=0$ ], in b quadriche ad invariante b-lineare nullo, i gruppi ${ }_{i}^{b}=0, \psi_{\lambda}^{h}=0$ sono coniugati. Onde si ricava pure: Dati due gruppi $w_{i=}^{b}=0, \psi_{i}^{b}=0$, se un $S_{b-\text { r }}$ tangente a tutte le quadriche di $\varphi_{\lambda}^{h}=0$ seca quelle di $\psi_{\lambda}^{h}=0$ in $b$ quadriche ad invariante b-lineare nullo, ogni altro $S_{h-\mathrm{r}}$ tangente a tutte le quadriche di $\varphi_{i}^{b}=0$ gode di questa proprietà, ed inoltre ogni $S_{h-1}$ tangente a tutte quelle di $\dot{\psi}_{\lambda}^{h}=0$ seca le quadriche di $\psi_{i}^{h}=0$ in $b$ quadriche ad invariante b-lineare nullo.

In particolate, come è ben noto: Se due coppie di quadriche di $[f]$ sidividono armonicamente, ogni retta tangente alle quadriche dell'una seca quelle dell'altra in due coppie di punti che si separano armonicamente. Ecc. ecc.

Se il gruppo $\varphi_{i}^{b}=0$ varia a descrivere un'involuzione $g_{h}^{r}(r<b)$ e si mantiene la condizione $(\varphi \psi)^{h}=0$, il gruppo $\psi_{i}^{b}=0$ varia a descrivere l'involuzione coniugata $g_{h}^{h-r-1}$. Se un $S_{h-1}$ tocca le quadriche di $u n$ gruppo della $g_{h}^{\prime}$, esso seca ogni gruppo della $g_{h}^{k-r-1}$ in $b$ quadriche ad invariante $b$-lineare nullo e reciprocamente. Di qui nasce una semplice generazione (per mezzo della $g_{b}^{\prime}$ ) della varietà degli $S_{h-1}$ secanti tutti i gruppi di una $\boldsymbol{g}_{b}^{b-r-1}$ in gruppi ad invariante $b$-lineare nullo ed in particolare $(r=h-1)$ del complesso degli $S_{h_{-1}}$ secanti un gruppo di $b$ quadriche in un gruppo ad invariante $b$ lineare nullo. Su tale complesso tornero più innanzi (vedi $n^{\circ}$ I 5 ); qui mi limito ad osservare che per $b=2$ si ottiene il complesso delle rette secanti due quadriche in coppie di punti separantisi armonicamente come costituito dai sistemi di tangenti comuni alle coppie di quadriche del fascio appartenenti all'involuzione ordinaria i cui ele-

*) Da quest'ultima osservazione si escluda il caso $n=\mathrm{I}$; per esso il fascio si riduce ad un ordinaria involuzione e l'annullarsi del discriminante di $\alpha_{\lambda}^{2}$ dà la condizione perchè l'involuzione sia parabolica (degenere). 
menti doppî sono le quadriche date. Per $n=3$ si ha cosi una nota generazione del complesso di Battaglini.

Come caso particolare di quanto si è svolto in principio del presente numero si ha: Se e $\varphi_{\mu}^{k} \varphi_{\nu}^{b-k}=0$, ogni $S_{b_{-1}}$ tangente alle $b$ quadriche $\varphi_{\lambda}^{b}=0 \sec a f_{\mu}$ ed $f_{\nu}$ in due quadriche di cui la prima è k-armonica alla seconda. Reciprocamente: Se un $S_{b-1}$ tangente alle $b$ quadriche $\varphi_{\lambda}^{h}=0$ seca $f_{\mu}$ ed $f_{\nu}$ in due quadriche di cui la prima e $k$-armonica alla seconda $\dot{e} \phi_{\mu}^{k} \varphi_{\nu}^{h-k}=0$. Segue che, se uno degli $S_{b-1}$ tangenti alle quadriche $\varphi_{\lambda}^{b}=0$ gode di tale proprietà, ne godono tutti gli altri.

Il significato ora stabilito per le forme polari permette di fissare anche quello di una qualunque spinta $(\varphi \psi)^{m} \varphi_{\lambda}^{h-m} \psi_{\lambda}^{l-m}$ di due forme $\varphi_{\lambda}^{b}, \psi_{\lambda}^{l}(b \leq n, l \leqslant n)$. Ed invero si fissi una $f_{\mu}$ di $[f]$; esistono $m$ quadriche di $[f]$ secate da ogni $S_{h-1}$ tangente alle $\varphi_{\lambda}^{h}=0$ in quadriche $m$-armoniche alla sezione di $f_{\mu}$ ed esse sono fornite dalla $\varphi_{\mu}^{h-m} \varphi_{\lambda}^{m}=0$; ne esistono $m$ secate da ogni $S_{l-\text { r }}$ tangente alle $\psi_{\lambda}^{l}=0$ in quadriche $m$-armoniche alla sezione di $f_{\mu}$ ed esse sono fornite dalla $\psi_{\mu}^{l-m} \psi_{\lambda}^{m}=0$. Condizione necessaria e sufficiente perchè ogni $S_{m-1}$ tangente alle prime $m$ quadriche [risp. alle seconde] tagli le seconde [risp. le prime] in $m$ quadriche ad invariante $m$-lineare nullo è appunto l'annullarsi di $(\varphi \psi)^{m} \varphi_{\mu}^{h-m} \psi_{\mu}^{l-m}$. Collo stesso metodo e più in generale si puó anche stabilire il significato di ogni formazione di tipo $\left(\psi_{\psi} \psi\right)^{m} \varphi_{\mu}^{b-m} \psi_{\nu}^{l-m}$. In particolare si puó invece supporre che $y$ coincida con $\psi$ e si ha cosi il significato delle spinte di una $\varphi_{\lambda}^{b}(b \leq n)$ su sè stessa.

Per quanto si è trovato al $\mathrm{n}^{\circ} 7$, il procedimento puó essere anche applicato alle spinte di $\alpha_{\lambda}^{n+1}$ su una $\psi_{\lambda}^{h}$ e su sè stessa. Così per es.: ogni quadrica $f_{\mu}$ ne determina in generale $2 s$ che le sono $2 s$-armoniche; condizione necessaria e sufficiente perchè ogni $S_{2 s-1}$ tangente alle $2 s$ quadriche le sechi in un gruppo di coni ad invariante $2 s$-lineare

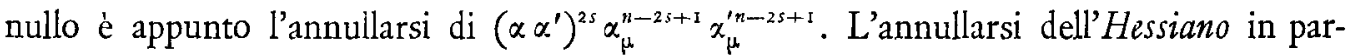
ticolare da la condizione perchè le due quadriche biarmoniche alla data coincidano.

Questa proprietà dell'Hessiano puó essere completata come segue.

Se $f_{\mu}$ appartiene al gruppo Hessiano la forma $\alpha_{\mu}^{n-1} \alpha_{\lambda}^{2}$ in $(\lambda)$ ha una radice doppia (v) ed $f_{\nu}$ appartiene al gruppo Steineriano.

Si ha cosi non solo $\alpha_{\mu}^{n-1} \alpha_{y}^{2}=0$, ma $\alpha_{\mu}^{n-1} \alpha_{y} \alpha_{\lambda} \equiv 0$ [id. in $(\lambda)$, quindi in particolare $\alpha_{\mu}^{n} \alpha_{\nu}=0$. Reciprocamente, dalle $\alpha_{\mu}^{n-1} \alpha_{\nu}^{2}=0, \alpha_{\mu}^{n} \alpha_{\nu}=0$ si risale all'ipotesi. Onde si ha: Se di due quadriche distinte $f_{\nu}, f_{\mu}$ la prima è insieme coniugata come luogo e biar. monica alla seconda, la prima appartiene al gruppo Steineriano del gruppo dei coni, mentre la seconda le corrisponde nel gruppo Hessiano; e reciprocamente. Non esistono nel fascio altre quadriche biarmoniche ad $f_{\mu}$.

9. Come conseguenza di quanto si è svolto in 8 si puó dedurre anche la seguente osservazione. Si ponga :

onde si ha:

$$
\varphi_{\lambda}^{n-k+1}=\alpha_{\mu}^{k} \alpha_{\lambda}^{n-k+1}
$$

$$
\varphi_{\mu}^{l} \varphi_{\rho}^{n-k-l+1}=\alpha_{\mu}^{k+l} \alpha_{\rho}^{n-k-l+1} .
$$

Colla precedente interpretazione: Se $f_{\mu} \dot{k}+$ l-armonica ad $f_{p}$, ogni $S_{n-k}$ tan- 
gente alle $n-k+\mathrm{I}$ quadriche di $[f]$ a cui $f_{\mu} \dot{e} k$-armonica, seca $f_{\mu}$ in una quadrica l-armonica alla sezione di $f_{p}$; e reciprocamente.

Il problema di determinare in un fascio le quadriche rispetto alle quali una data è $h$-armonica è cosi ridotto alla ripetizione dell'altro: determinare in un fascio le quadriche rispetto alle quali una data è coniugata come luogo, ed alla ricerca degli $S_{r \rightarrow \text { s }}$ tangenti ad $r$ quadriche. Ed invero, trovate in $[f]$ le $n$ quadriche rispetto a cui $f_{\mu}$ è coniugata come luogo, si tagli $[f]$ con un $S_{n-1}$ tangente a queste; nel fascio $\left[f^{\prime}\right]$ sezione le $(n-\mathrm{I})$ quadriche a cui $f_{\mu}^{\prime}$ è coniugata come luogo sono sezione delle $(n-\mathrm{I})$ di $[f]$ alle quali $f_{\mu}$ è biarmonica; si tagli $[f]$ con un $S_{n-2}$ tangente a queste ultime; nel fascio $\left[f^{\prime \prime}\right]$ sezione le $(n-2)$ quadriche a cui $f_{\mu}^{\prime \prime}$ è coniugata come luogo sono sezione delle $(n-2)$ di $[f]$ alle quali $f_{\mu}$ è 3 -armonica ecc. ecc.

Più in generale ed in modo del tutto analogo si potrebbe determinare la quadrica del fascio che con $n$ date di esso formi un gruppo ad invariante $(n+\mathrm{I})$-lineare nullo.

19. Introduco le $n+\mathrm{I}$ formazioni :

$$
\begin{aligned}
& \alpha_{\lambda}^{n+1}=\left(a_{\lambda}^{\prime} a_{\lambda}^{\prime \prime} \ldots a_{\lambda}^{(n+1)}\right)^{2} \\
& \beta_{\lambda}^{n}=\left(a_{\lambda}^{\prime} a_{\lambda}^{\prime \prime} \ldots a_{\lambda}^{(n)} u\right)^{2} \\
& \theta_{\lambda}^{k}=\left(a_{\lambda}^{\prime} a_{\lambda}^{\prime \prime} \ldots a_{\lambda}^{(k)} u^{\prime} u^{\prime \prime} \ldots u^{(p-k+\mathrm{s})}\right)^{2} \\
& \tau_{\lambda}^{2}=\left(a_{\lambda}^{\prime} a_{\lambda}^{\prime \prime} u^{\prime} u^{\prime \prime} \ldots u^{(n-1)}\right)^{2} \\
& \omega_{\lambda}=\left(a_{\lambda} u^{\prime} u^{\prime \prime} \ldots u^{(n)}\right)^{2}
\end{aligned}
$$

che dico combinanti binari elementari del fascio.

Il primo di essi è il discriminante, ed è noto per quanto precede. I rimanenti (vedi $n^{\circ}$ I) tenuto fisso $(\lambda)$ rappresentano, col loro annullarsi, $f_{\lambda}$ ordinatamente come inviluppo di iperpiani, ... come complesso degli $S_{k-1}$ tangenti, ... come complesso delle rette tangenti, come luogo di punti. Gli stessi, tenute fisse le $u$, rappresentano, col loro annullarsi, ordinatamente $\mathrm{i}$ gruppi formati dalle quadriche di $[f]$ tangenti all' $S_{n-1}$ di coordinate $u_{i}, \ldots$ all'S $S_{k-1}$ intersezione degli iperpiani di coordinate $u_{i}^{(h)}(b=\mathrm{I}, 2, \ldots n-k+\mathrm{I}), \ldots$ alla retta intersezione degli iperpiani di coordinate $u_{i}^{(b)}(b=\mathrm{I}, 2, \ldots n-\mathrm{I})$, infine quello formato dalla quadrica di $[f]$ passante per il punto intersezione degli iperpiani di coordinate $u_{i}^{(h)}(b=\mathrm{I}, 2, \ldots n)$.

L'identico annullarsi di $\theta_{\lambda}^{k}(2 \leq k \leq n+\mathrm{I})$ disgiunto dall'identico annullarsi dei seguenti combinanti elementari è condizione necessaria e sufficiente perchè la quadrica generica del fascio sia $n-k+2$ volte specializzata.

Ogni forma invariantiva simultanea di $\beta_{\lambda}^{n}$ e di $\alpha_{\lambda}^{n+1}$, di grado $m$ nei coefficienti di $\beta_{\lambda}^{n}$ rappresenta col suo annullarsi un inviluppo di classe $2 m$, annesso invariantivamente ad $f_{\lambda}$ nel caso di un covariante binario ed annesso invariantivamente al fascio nel caso di un irvariante binario. L'annullarsi identico di un covariante rappresenta in generale una varietd di iperpiani annessa invariantivamente al fascio.

Più in generale, ogni forma invariantiva simultanea di $\theta_{\lambda}^{k}$ e di $\alpha_{\lambda}^{n+\mathrm{r}}$, di grado $m$ nei coefficienti di $\theta_{k}^{k}$ rappresenta col suo annullarsi un complesso di spazii $S_{k-1}$ di grado 
$2 \mathrm{~m}$, annesso invariantivamente ad $f_{\lambda}$ o ad $[f]$ secondo che si tratti di covariante o di invariante binario; l'annullarsi identico di un covariante rappresenta in generale una varietà di spazii $S_{k-1}$ annessa invariantivamente al fascio.

Se nella formazione invariantiva oltre alle $\alpha_{\lambda}^{n+1}, \theta_{\lambda}^{k}$ si introduce anche una binaria $\varphi_{h}^{h}$, l'invariantività della varietd rappresentata è relativa al gruppo di quadriche $\varphi_{\lambda}^{h}=0$.

Le formazioni in cui oltre ad $x_{\lambda}^{n+1}$ entri più di un combinante elementare condurrebbero alla considerazione di connessi e di corrispondenze.

Non è peró vero reciprocamente che ogni variet legata invariantivamente al fascio o ad un gruppo di quadriche del fascio sia sempre suscettibile di una rappresentazioae del tipo ora considerato. Basti osservare che una forma invariantiva contenente $i$ coefficienti di $\omega_{\lambda}$ rappresenta col suo annullarsi il luogo spezzantesi nelle quadriche del fascio appartenenti al gruppo rappresentato dall'annullarsi della stessa formazione in cui al simbolo $\omega$ si sia sostituita la variabile binaria corrente. Ora è ben nota l'esistenza di luoghi legati invariantivamente al fascio e non spezzantisi in quadriche di esso. E per altro evidente che i combinanti elementari e le loro formazioni invariantive occupano un posto notevole nella geometria del fascio di quadriche.

Dal teorema col quale si chiude il $\mathbf{n}^{\circ} 7$ e dal significato dei combinanti elementari si deducono gli enunciati :

Il discriminante di $\beta_{\lambda}^{*}$, considerata come forma binaria, rappresenta col suo annullarsi l'inviluppo degli iperpiani tangenti alla quartica base.

Il discriminante di $\mathrm{G}_{2}^{k}(2<k<n)$, considerata come forma binaria, rappresenta col suo annullarsi il complesso degli $S_{k_{-1}}$ tangenti alla quartica base (cioè secanti la quartica base in una varietà dotata di punto doppio).

Il discriminante di $\tau_{\lambda}^{2}$, considerata come forma binaria, rappresenta col suo annullarsi il complesso delle rette secanti la quartica base.

Dallo stesso significato dei combinanti elementari si deduce che lidentico annullarsi, rispetto a $(\lambda)$, di $\theta_{\lambda}^{k}(k>2)$ caratteri $i_{2}$ a gli $S_{k-1}$ secanti il fascio in un fascio di quadriche tutte specializzate, cioè gli $S_{k-1}$ tangenti a tutte le quadriche del fascio, e che l'identico annullarsi, rispetto a $(\lambda)$, di $\tau_{\lambda}^{2}$ caratterizza le rette tangenti alla quartica base. Cosi l'identico annullarsi, rispetto a $(\lambda)$, di $\omega_{\lambda}$ caratterizza $i$ punti della quartica base. Segue che ogni varietà rappresentata dall'annullarsi di una forma invariantiva di $\theta_{\lambda}^{k}$ contiene gli $S_{k-1}$ tangenti a tutte le quadriche del fascio, che ogni varieta, in particolare, rappresentata dall'annullarsi di una forma invariantiva di $\tau_{\lambda}^{2}$ contiene quella delle tangenti alla quartica base. Il risultato analogo per $\omega_{\lambda}$ è già compreso in una precedente osservazione.

Altra considerazione d'indole generale è la seguente: Se $\varphi_{\lambda}^{r} \dot{e}$ una forma binaria, il risultante di $\varphi_{\lambda}^{r}$ e di $\theta_{\lambda}^{k}\left(d i \beta_{\lambda}^{n}\right.$, di $\left.\omega_{2}\right)$ col suo annullarsi fornisce l'equazione complessiva delle quadriche del gruppo $\varphi_{\lambda}^{r}=0$ considerate come complesso degli $S_{k-1}$ tangenti (come inviluppo di iperpiani, come luogo di punti).

Cosi : L'identico annullarsi rispetto alle variabili contragredienti del risultante di $\alpha_{\lambda}^{n+1}$ e del combinante $\theta_{\lambda}^{k}$, senza la proprietà analoga per il combinante elementare suc- 
cessivo è condizione necessaria e sufficiente per l'esistenza di (almeno) una quadrica di $[f]$ $n-k+2$ volte specializzata, senza quella di quadriche specializzate piu di $n-k+2$ volte $\left.{ }^{*}\right)$. Ecc. ecc.

\section{$\int$ 3. Gli inviluppi di seconda classe $(\Phi(\%))^{n}=0$}

I1. Da quanto si è veduto al $\mathrm{n}^{\circ} 7$ (od anche al $\mathrm{n}^{\circ} 8$ ) risulta ora in modo semplice: Gli iperpiani secanti $n$ quadriche $f_{\mu}, f_{\nu}, \ldots f_{\rho} d i[f]$ in $n$ quadriche ad invariante $n$-lineare nullo costituiscono l'inviluppo di $2^{\text {a }}$ classe di equazione:

$$
\beta_{\mu} \beta_{\nu} \ldots \beta_{\rho}=0 \text {. }
$$

La (I) per il teorema del $n^{\circ} 6$ si puó scrivere anche cosi :

$$
\left(a_{\mu} a_{\nu} \ldots a_{p} u\right)^{2}=0 .
$$

La stessa (I), qualora si ponga : prende l'aspetto :

$$
\Phi_{\lambda}^{n}=(\mu \lambda)(\nu \lambda) \ldots(\rho \lambda)
$$

$$
\left(\Phi(\beta)^{n}=0\right.
$$

Se $\Phi_{\lambda}^{n}$ si considera come forma parametrica, risulta che l'inviluppo (4) descrive un sistema lineare. Escluso il caso in cui ogni quadrica di $[f]$ sia almeno due volte specializzata, pel quale (4) è sempre indeterminato, quando per $\boldsymbol{\Phi}_{\lambda}^{n}$ si prenda una forma generica, l'inviluppo (4) è ben determinato, poichè è tale quando per $\boldsymbol{\Phi}_{\lambda}^{n}$ si prenda la potenza $n$-esima di una forma lineare generica. Risulta dunque che (quando non si tenga conto degli inviluppi eventualmente indeterminati) il sistema (4) è al più $\infty^{n}$.

Esso è effettivamente $\infty^{n}$ quando il fascio non sia dotato di quadriche specializzate due o più volte. Infatti in tal caso $n$ iperpiani generici determinano come rispettivi combinanti $\omega_{\text {, }}^{n}$ altrettante forme generiche, le quali individuano la forma $\boldsymbol{\Phi}_{\lambda}^{n}$ ad esse conjugata e quindi l'inviluppo $(\Phi \xi)^{n}=0$. Se invece $[f]$ contiene $r$ quadriche specializzate più di una volta, corrispondenti ai fattori lineari $q_{\lambda}^{\prime}, q_{\lambda}^{\prime \prime}, \ldots q_{\lambda}^{(r)}$ e se nel fascio sezione di $[f]$ con un $S_{n-1}$ generico le loro sezioni contano rispettivamente $s_{1}, s_{2}, \ldots s_{r}$ volte nel gruppo delle quadriche specializzate, il combinante $\beta_{\lambda}^{n}$ di un iperpiano generico contiene come fattore:

$$
\Psi=\Psi_{\lambda}^{\Sigma_{s_{i}}}=q_{\lambda}^{\prime s_{1}} q_{\lambda}^{\prime \prime s_{2}} \ldots q_{\lambda}^{(r) s_{r}} .
$$

Segue che $\beta_{\lambda}^{n}$, quando non sia indeterminato, varia in un sistema lineare $n-\sum_{i}^{r} s_{i}$ volte infinito. Assunti $n-\sum_{i}^{r} s_{i}$ iperpiani generici, questi determinano come rispettivi combinanti $\rho_{\lambda}^{n}$ altrettante forme generiche del sistema, quindi il sistema $\infty^{\Sigma_{s_{i}}}$ delle forme $\cdot \Phi_{\lambda}^{n}$ ad esse conjugate. Ma ciascuna di tali $\infty^{\Sigma_{s_{i}}}$ forme $\Phi_{\lambda}^{n}$ conduce sempre ad un medesimo inviluppo (4); onde il sistema (4), esclusi gli inviluppi indeterminati, è $\infty^{n-\Sigma s_{i}}$.

*) Per quest'ultimo teorema si supponga $\alpha_{\lambda}^{n+1} \neq \equiv$. 
L'indeterminazione nasce quando $\Phi_{i}^{n}$ è coniugata a tutte le forme $\epsilon_{i}^{n}$, ossia quando $\Psi$ è apolare rispetto a $\Phi_{\lambda}^{n}$.

Le precedenti considerazioni possono essere chiarite anche osservando che, nell'ipotesi più generale, ogni forma $\Phi_{\lambda}^{n}$ si puó ridurre al tipo ${ }^{*}$ ):

$$
\Phi_{\lambda}^{n}=\sum_{i=1}^{r} \delta_{\lambda}^{(i)^{s_{i}-1}} q_{\lambda}^{(i)^{n-s_{i}+1}}+\sum_{j=1}^{m} d^{(j)} \xi_{\lambda}^{(j)^{n}}
$$

dove le $\delta_{\lambda}^{(i)^{s^{i}-1}}$ sono forme parametriche da prendersi in tutti $\mathrm{i}$ modi possibili, le $d^{(j)}$ costanti arbitrarie, le $c_{\lambda}^{\prime j}$ fattori lineari distinti fissati comunque purchè diversi dai fattori $q_{\lambda}^{(i)}$, infine è $m=n-\sum_{i=1}^{r} s_{i}+$ I. Se ne deduce, tenute presenti le

$$
\left.\left(\beta q^{(i)}\right)^{n-s_{i}+1} \beta_{\lambda}^{s_{i}-1} \equiv 0 \quad \text { [ident. risp. a }(\hat{\lambda})\right]
$$

sussistenti in base alle ipotesi fatte, anche:

in armonia con quanto precede.

$$
(\Phi \beta)^{n}=\sum_{j=1}^{m} d^{(j)}\left(\xi^{(j)} \beta\right)^{n}
$$

Concludendo :

Il sistema $(\Phi \beta)^{n}=$ o è completamente indeterminato quando, e solo quando, tutte le quadriche di $[f]$ sono almeno due volte specializzate. In ogni altro caso, se le $r \gg 0$ quadriche di [f] specializzate piu di una volta sono secate da un $S_{n-1}$ generico in quadriche da contarsi $s_{1}, s_{2}, \ldots s_{r}$ volte nel gruppo dei coni del fascio sezione, il sistema $(\Phi \beta)^{n}=0 \dot{e} \infty^{n-\Sigma s_{i}}$, salvo l'indeterminazione dell'inviluppo per le forme $\Phi_{\grave{\lambda}}^{n}$ a cui $\dot{e}$ apolare il gruppo delle quadriche piu volte specializzate contate rispettivamente $s_{1}, s_{2}, \ldots s_{r}$ volte.

Non credo inopportuno porre in luce come dall'ultima parte del precedente enunciato risulti in $S_{n}$ l'esistenza di gruppi di $n$ quadriche secate da ogni iperpiano in $n$ quadriche ad invariante $n$-lineare nuilo, anzi, colle notazioni usate, l'esistenza di $\infty^{\Sigma_{s_{i}-1}}$ gruppi di tal natura in $[f]$ (se $r>0)$.

Lo stesso enunciato puó assumere una forma più espressiva nel caso di un fascio di quadriche non tutte specializzate. Introdotti, secondo WeIERSTRass, gli esponenti dei divisori elementari, si supponga il fascio caratterizzato dal simbolo di SEGRE **):

$$
\left[\left(e_{1}, e_{1}^{\prime}, \ldots e_{1}^{\left(b_{1}-1\right)}\right)\left(e_{2}, e_{2}^{\prime}, \ldots e_{2}^{\left(h_{2}-1\right)}\right) \ldots\left(e_{t}, e_{t}^{\prime}, \ldots e_{i}^{\left(h_{t}-1\right)}\right)\right] .
$$

Da note formole $\left.{ }^{* * *}\right)$ si deduce immediatamente:

onde anche:

$$
\sum_{i=1}^{r} s_{i}=n+\mathrm{I}-\sum_{j=1}^{t} e_{j},
$$

$$
n-\sum_{i=1}^{r} s_{i}=\sum_{j=1}^{t} e_{j}-\mathrm{I} .
$$

*) Il primo sommatorio fornisce una forma generica per cui sia apolare $\Psi$.

*) SEgRe, Studio sulle quadriche, etc. (cit.), Parte $2^{\mathrm{a}}, \S 3, \mathrm{n}^{\mathrm{o}} 63$.

***) WeIERSTRAss, Zur Theorie der bilinearen und quadratischen Formen (cit.), $\mathrm{n}^{\circ} \mathrm{I}$, form. (4). Cfr. Segre, loc. cit. 
Si ha cosi: Per un fasio di quadriche non tutte specialižate, la somma dei primi esponenti dei singoli gruppi di divisori elementari fornisce il massimo numero di inviluppi ben determinati e linearmente indipendenti che $\dot{e}$ possibile assumere in $(\Phi){ }^{n}=0$.

12. Se si osserva che ogni $\boldsymbol{\Phi}_{\lambda}^{n}$ si puó esprimere come combinazione lineare di potenze $n$-esime di forme lineari, risulta: Il sistema $(\Phi \beta)^{n}=0$ l il sistema lineare al quale "appartiene» quello algebrico delle quadriche di $[f]$ pensate come inviluppo, escluse quelle indeterminate come tali. Questa considerazione, notevole per sè stessa, puó essere applicata per es. alla determinazione di tutti i tipi di fascio-scbiera. Si noti perció anzitutto che un fascio di coni non è in alcun caso fascio-schiera, come si puó stabilire direttamente in modo semplice. Segue che, tenuto presente l'enunciato col quale si chiude il $\mathrm{n}^{\circ}$ I $\mathrm{I}$, i fasci-schiera sono quelli di quadriche non tutte specializzate soddisfacenti alla $\sum_{j=1}^{t} e_{j}=2$.

Questa conduce od a : $t=\mathrm{I}, e_{1}=2$ oppure a: $t=2, e_{\mathrm{x}}=e_{2}=\mathrm{I}$; onde si ha: Ogni fascio-schiera si riduce ad uno dei tipi:

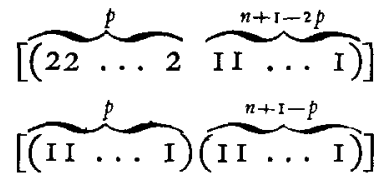

$$
\begin{gathered}
(0<2 p \leq n+\mathrm{I}) \\
(0<p<n+\mathrm{I})
\end{gathered}
$$

Alcuni dei precedenti sviluppi acquistano maggiore evidenza quando si rappresentino gli inviluppi di $2^{2}$ classe di $S_{n}$ coi punti di uno spazio $R$ ad $\frac{n(n+3)}{2}$ dimensioni. Se $[f]$ è nelle condizioni generali del $\mathrm{n}^{\circ} \mathrm{I} \mathrm{I}$, esistono $n-\sum_{i=1}^{r} s_{i}$ quadriche variabili di esso che, come inviluppo, soddisfano alla condizione lineare di toccare un $S_{n-1}$ generico di $S_{n}{ }^{*}$ ); e poichè ogni condizione lineare a cui soddisfi un inviluppo di seconda classe è combinazione lineare di condizioni del tipo ora detto, risulta che più in generale esistono $n-\sum_{i=1}^{r} s_{i}$ quadriche variabili di $[f]$ che soddisfino, come inviluppo, ad una generica condizione lineare. Segue che, nella rappresentazione di cui sopra, le quadriche di $[f]$, considerate come inviluppo, quando si escludano quelle indeterminate come tali, sono rappresentate da punti di una curva razionale di ordine $n-\sum_{i=1}^{r} s_{i}$. Essa è curva normale dello spario $R^{\prime}$ ad $n-\sum_{i=1}^{r} s_{1}$ dimensioni $i$ cui punti rappresentano gli inviluppi $\left.(\Phi) *\right)^{n}=0$ non indeterminati.

La rappresentazione accennata è semplicemente realizzabile nel caso di un fascio di quadriche non tutte specializzate, quando si costruiscano i poli di un $S_{n-1}$ generico rispetto alle quadriche di $[f]$, escluse quelle più volte specializzate ed in generale rispetto agli inviluppi $(\Phi \beta)^{n}=0$, esclusi quelli indeterminati. Si ha cosi il seguente teorema,

*) Se il fascio non è di quadriche tutte specializzate ne esistono $\sum_{j=1}^{t} e_{j}-\mathrm{I}$. [SEGRE, Studio sulle quadriche, etc. (cit.), Parte $\left.2^{\mathrm{a}}, \S 3, \mathrm{n}^{\circ} 75\right]$ in accordo col risultato in fine del $\mathrm{n}^{\circ}$ I r. 
che per un fascio privo di quadriche più volte specializzate coincide con uno del Prof. SEGRE ${ }^{*}$ ):

$S e[f]$ non è di quadriche tutte specializzate, $i$ poli di un $S_{n-1}$ generico rispetto alle quadriche di $[f]$ non pii volte specializxate giacciono sopra una curva razionale d'ordine $n-\sum_{i=1}^{r} s_{i}=\sum_{j=1}^{t} e_{j}$ - I appartenente ad uno spazio ad $n-\sum_{i=1}^{r} s_{i}$ dimensioni.

Nel caso di un fascio di quadriche tutte specializzate (una volta) il teorema del $\mathrm{n}^{0}$ I I sulla dimensione del sistema $(\boldsymbol{\Phi} \beta)^{n}=\mathrm{o}$ puó ricevere una semplice conferma. In questo caso ${ }^{* *}$ ) il luogo dei vertici è una curva $\Gamma_{l}$ razionale di ordine $l \supseteq 0$ appartenente ad un $S_{l}$ ed il sistema $\left(\Phi()^{n}=0\right.$, come risulta da una precedente osservazione $\left.{ }^{* * *}\right)$, è quello degli inviluppi conjugati a tutte le quadriche passanti per $\boldsymbol{\Gamma}_{l}$. Ora, poichè il passaggio per $\Gamma_{l}$ impone ad una quadrica $2 l+\mathrm{I}$ condizioni lineari, risulta che $(\boldsymbol{\Phi} \beta)^{n}=0$ è $\infty^{2 l} \dagger$ ). Ma allo stesso risultato si perviene col metodo del $n^{\circ}$ I I, quando si noti $+f$ ) che il fascio generale di quadriche una volta specializzate $\mathrm{i}$ cui vertici sono punti di una $\Gamma_{l}$ possiede $n-2 l$ quadriche due volte specializzate, ciascuna delle quali è secata da un $S_{n-1}$ generico in una quadrica una volta specializzata.

13. Dal $\mathrm{n}^{0}$ I I risulta che ad un gruppo $\Phi_{\lambda}^{n}=0$ di $n$ quadriche, non appartenente alla $g_{n}^{\Sigma_{s_{i}-1}}$ dei gruppi pei quali è apolare $\Psi$, corrisponde un inviluppo ben determinato $\left(\Phi(s)^{n}=0\right.$.

Ad un inviluppo $(\Phi \beta)^{n}=0$ corrispondono invece, insieme al gruppo $\Phi_{\lambda}^{n}=0$, tutti i gruppi della $g_{n}^{\Sigma_{s_{i}}}$ individuata dalla $g_{n}^{\Sigma_{s_{i}-1}}$ singolare e dallo stesso gruppo $\Phi_{\lambda}^{n}=0$. Segue :

Ai gruppi di una $g_{n}^{d}$ non avente colla $g_{n}^{\Sigma_{s_{i-1}}}$ singolare alcun gruppo in comune corrispondono gli inviluppi $(\Phi \beta)^{n}=0$ di un sistema lineare $\infty^{d}$.

Ai gruppi di una $g_{n}^{d}$ avente in comune colla $g_{n}^{\Sigma_{s_{i}-1}}$ singolare una $g_{n}^{p}$ corrispondono gli inviluppi $\left(\Phi(s)^{n}=0\right.$ di un sistema lineare $\infty^{d-p-\mathrm{T}}$.

Quindi:

Ai gruppi di una $g_{n}^{\prime \prime}$ generica per $d \leq n-\sum s_{i}$ corrispondono gli inviluppi di un sistema lineare $\infty^{d}$, per $d \supseteq n-\sum s_{i}$ gli inviluppi dell'intero sistema $\infty^{n-\Sigma_{s_{i}}}$ (il caso $d=n-\sum s_{i}$ essendo cosi compreso nei due enunciati).

Se infine $[f]$ non contiene quadriche specializzate più di una volta $\left(\sum s_{i}=0\right)$, ai gruppi di ogni $g_{n}^{d}$ corrispondono gli inviluppi di un sistema $\infty^{d}$.

Considero in particolare la $g_{n}^{n-2}$ dei gruppi apolari al gruppo degli $n+$ I coni

*) Studio sulle quadriche, etc. (cit.), Parte $2^{\mathrm{a}}, \S \mathrm{I}, \mathrm{n}^{\mathrm{o}} 55$.

**) v. Segre, Ricerche sui fasci di coni quadrici, etc. (cit.), $\mathrm{n}^{\mathrm{o}} 7$.

***) Al no $4, \beta$ ) per $k=n$.

†) Si puó aggiungere che gli inviluppi $(\Phi \beta)^{n}=0$ hanno per nuclei le quadriche di $S_{l}$, coniugate come inviluppo a quelle pure di $S_{l}$ passanti per $\Gamma_{l}$.

t†) v. Segre, Ricerche sui fasci di coni quadrici, etc. (cit.), $\mathrm{n}^{\circ} \mathrm{I} 6$.

Rond. Circ. Matew. Palermo, t. XXIII (1907). - Stampato il 4 aprile 1907. 
$\left(\alpha_{\lambda}^{n+1}=0\right)$, supposto $[f]$ composto di quadriche non tutte specializzate $\left.{ }^{*}\right)$. Dico che tale $g_{n}^{n-2}$ contiene come subordinata la $g_{n}^{\Sigma_{s_{i}-1}}$ singolare. Per questo osservo dapprima che posto, come al $\mathrm{n}^{\mathrm{o}}$ I I : $\Psi=q_{\lambda}^{s_{s}} q_{\lambda}^{\prime s_{2}} \ldots q_{\lambda}^{(r) s_{r}}$, la $\alpha_{\lambda}^{n+1}$ contiene come fattore $\Psi^{*}=q_{\lambda}^{\prime s^{+1}} q_{\lambda}^{\prime \prime s_{2}+1} \ldots q_{\lambda}^{(\gamma) s_{r}+\mathbf{r}}$.

Ed invero se $f_{\mu}^{\prime}$, sezione di $f_{\mu}$ con un $S_{n-1}$ generico, è da contarsi $s$ volte nel gruppo dei coni del fascio sezione, si ha:

$$
\beta_{\mu}^{n-s+1} \beta_{\lambda}^{s-1} \equiv 0 \quad[\text { id. rispetto a }(\lambda)],
$$

ossia, qualunque siano le $u$, tenuto presente il teorema del $\mathrm{n}^{\circ} 6$ :

$$
\left(a_{\mu}^{\prime} a_{\mu}^{\prime \prime} \ldots a_{\mu}^{(n-s+1)} a_{\lambda}^{\prime} a_{\lambda}^{\prime \prime} \ldots a_{\lambda}^{(s-1)} u\right)^{2} \equiv 0 .
$$

Sostituendo alle variabili $u$ i simboli $a_{\lambda}^{(s)}$, si deduce:

ossia :

$$
\left(a_{\mu}^{\prime} a_{\mu}^{\prime \prime} \ldots a_{\mu}^{(n-s+1)} a_{\lambda}^{\prime} a_{\lambda}^{\prime \prime} \ldots a_{\lambda}^{(s-1)} a_{\lambda}^{(s)}\right)^{2} \equiv 0,
$$

$$
\alpha_{\mu}^{n-s+1} \alpha_{\lambda}^{s} \equiv 0 \text {, }
$$

la quale dimostra che $f_{\mu}$ è da contarsi almeno $s+$ I volte fra $i$ coni di $[f]$.

Essendo cosi provato che $\alpha_{\lambda}^{n+1}$ contiene come fattore $\Psi^{*}$, se ne deduce che $(x \Phi)^{n} \alpha_{2}$ possiede il fattore simbolico $(\Psi \Phi)^{\Sigma_{s_{i}}}$. Quindi, se $\Psi$ è apolare a $\boldsymbol{\Phi}_{\lambda}^{n}\left[(\Psi \Phi)^{\Sigma_{s_{i}}} \Phi_{\lambda}^{n-\Sigma_{s_{i}}} \equiv \mathrm{o}\right]$, è pure $\Phi_{\lambda}^{n}$ apolare ad $\alpha_{\lambda}^{n+\mathrm{r}}\left[(\alpha \Phi)^{n} x_{\lambda} \equiv \mathrm{o}\right]$, come appunto si voleva dimostrare.

$\mathrm{Dal}$ criterio generale precedentemente esposto si ricava cosi:

Alla $g_{n}^{n-2}$ dei gruppi apolari a quello degli $n+\mathrm{I}$ coni corrisponde un sistema lineare $\infty^{n-\Sigma_{s_{i}-2}}$ di inviluppi $(\Phi \beta)^{n}=0$.

Per stabilire il significato di questo sistema si osservi che, posto, come altrove, $\Phi_{\lambda}^{n}=(\mu \lambda)(\nu \lambda) \ldots(p \lambda)$, la

$$
(\zeta)=(\alpha \Phi)^{n} \alpha_{\lambda}=0
$$

per il teorema già citato del $\mathrm{n}^{0} 6$, si puó scrivere: cioè si deduce dall'equazione :

$$
\left(a_{\mu} a_{\nu} \ldots a_{\rho} a_{\lambda}\right)^{2}=0
$$

$$
\left(a_{\mu} a_{v} \ldots a_{p} u\right)^{2}=0
$$

di $(\Phi \beta)^{n}=0$, sostituendo alle variabili $u$ i simboli $a_{\lambda}$. Segue che, indicando con $h_{x}^{2} \equiv h_{x}^{\prime 2} \equiv \cdots=$ o l'equazione locale di $(\Phi \beta)^{n}=0$, la (7) si identifica con:

$$
\left(b^{\prime} b^{\prime \prime} \ldots b^{(n)} u\right)^{2}=0
$$

e quindi la (6), ossia la (5), con:

$$
\left(b^{\prime} b^{\prime \prime} \ldots b^{(n)} a_{\lambda}\right)^{2}=0 .
$$

Si giunge così intanto ad un notevole risultato che puó essere enunciato in uno dei seguenti modi :

1) L'inviluppo $(\Phi \beta)^{n}=0$ è coniugato ad una, ed in generale ad una sola, quadrica $d i[f]$, il cui parametro annulla la spinta $(\alpha \Phi)^{n} \alpha_{\lambda}$.

*) La $g_{n}^{n-2}$ degenera in una $g_{n}^{n-1}$ quando, e solo quando, $x_{\lambda}^{n+1}$ è una potenza di forma lineare. In questo caso alcune delle cose che seguono debbono subire qualche modificazione. 
2) Se il gruppo $\Phi_{i}^{n}=0$ è, nel campo binario, coniugato al gruppo delle $n$ quadriche $d i[f]$ coningate come inviluppo ad $f_{\lambda}$, e solo in tal caso, l'inviluppo $(\Phi \beta)^{n}=0$ è coniugato ad $f_{\lambda}$.

3) Se $n+\mathrm{I}$ quadriche di $[f]$ sono ad invariante $(n+\mathrm{I})$-lineare nullo, l'inviluppo degli $S_{n-1}$ secanti $n$ di queste in $n$ quadriche ad invariante n-lineare nullo è coniugato alla rimanente; e reciprocamente.

$\mathrm{Ma}$ il caso in cui ( 5 ) sussista identicamente conduce al significato del sistema $\infty^{n-\Sigma_{s_{i}-2}}$ in questione. Risulta infatti :

Condizione necessaria e sufficiente, perchè l'inviluppo $(\Phi \beta)^{n}=0$ sia coniugato a tutte le quadriche del fascio [f], è che sia $\Phi_{\lambda}^{n}$ apolare ad $\alpha_{\lambda}^{n+1}$. Gli inviluppi del sistema sono dunque tutti e solo quelli coniugati ad ogni quadrica del fascio [e appartenenti al sistema $\left.\left.(\Phi \beta)^{n}=0\right]^{*}\right)$.

Nel campo binario del fascio, alla $g_{n}^{n-2}$ dei gruppi apolari a quello dei coni è coniugata la $g_{n}^{\prime}$ dei gruppi $\alpha_{\sigma} \alpha_{\lambda}^{n}=0$ polari del gruppo stesso ${ }^{* *}$ ). Alla $g_{n}^{\prime}$ corrisponde in generale una schiera di inviluppi

$$
\alpha_{\sigma}(\alpha \beta)^{n}=0,
$$

che diro schiera associata al fascio $[f]^{* * *}$, riferita projettivamente a questo, corrispondendosi l'inriluppo (8) e la quadrica $f_{\sigma}$. L'inviluppo (8) puó essere definito come quello degli $S_{n-1}$ secanti in $n$ quadriche ad invariante n-lineare nullo le $n$ quadriche di $[f]$ coniugate come inviluppo ad $\left.f_{\sigma}^{* * * *}\right)$.

Per $n$ dispari si ha:

$$
\left(\alpha \alpha^{\prime}\right)^{n} \alpha_{\sigma} \alpha_{\lambda}^{\prime}=\frac{1}{2}\left(\alpha \alpha^{\prime}\right)^{n}\left(\alpha_{\sigma} \alpha_{\lambda}^{\prime}-\alpha_{\lambda} \alpha_{\sigma}^{\prime}\right)=\frac{1}{2}\left(\alpha \alpha^{\prime}\right)^{n+1}(\sigma \lambda)
$$

onde, ricordando l'enunciato I) del presente $\mathrm{n}^{\circ}$ :

Per $n$ dispari in generale ciascuna quadrica del fascio è coniugata all'inviluppo corrispondente della schiera associata e a nessun altro di questa; se pero il gruppo dei coni $\dot{e}$ ad invariante $\left(n+\mathrm{I}\right.$ )-lineare nullo (cfr. $\left.\mathrm{n}^{\circ} 7\right)$, ogni quadrica di $[f]$ è coniugata a tutti gli inviluppi (8).

Per $n$ pari, posto:

$$
\left(\alpha \alpha^{\prime}\right)^{n} x_{\lambda} \alpha_{\lambda}^{\prime}=\Delta_{\lambda}^{2}
$$

*) Nel caso già citato in nota in cui la $g_{n}^{n-2}$ degenera in una $g_{n}^{n-1}$, il sistema degenera in uno $\infty^{n-\Sigma s_{i}-1}$. Ciò si spiega anche notando che l'unica quadrica specializzata è come luogo coniugata a tutte le altre di $[f]$ (v. $\mathrm{n}^{\circ} 7$ ), quindi a tutti gli inviluppi $(\Phi \beta)^{n}=0$.

${ }^{* *}$ ) Nel caso della precedente nota la $g_{n}^{\prime}$ si riduce ad un gruppo, salvo l'indeterminazione per $f_{\sigma}$ coincidente coll'unica quadrica specializzata.

$\left.{ }^{* * *}\right)$ La scelta della denominazione mi è suggerita dal fatto che la schiera associata ad un fascio di coniche generico ha per quadrilatero base il quadrilutero associato al quadrangolo base del fascio, nel senso di КонN [Ueber das Vierseit und sein associirtes Viereck, das Fünflach und sein associirtes Fünfeck; Sitzungsber. d. Wien. Akad., Bd. XCIII (I886), pp. 314-352], come avro occasione di dimostrare in altro lavoro.

****) L'inviluppo (8) è pure quello degli $S_{n-1}$ tangenti ad $n$ quadriche di $[f]$ formanti con $f_{\sigma}$ un gruppo ad invariante $(n+1)$-lineare nullo. 
si ha:

ossia :

$$
\left(\alpha \alpha^{\prime}\right)^{n} \alpha_{\sigma} \alpha_{\lambda}^{\prime}=\frac{1}{2}\left(\alpha \alpha^{\prime}\right)^{n}\left(\alpha_{\sigma} \alpha_{\lambda}^{\prime}+\alpha_{\lambda} \alpha_{\sigma}^{\prime}\right)=\Delta_{\sigma} \Delta_{\lambda}
$$

Per $n$ pari in $[f]$ è in generale determinata un'involuzione ordinaria $\Delta_{\sigma} \Delta_{\lambda}=0$, in modo che, di due quadriche di uno stesso gruppo, ciascuna è coniugata all'inviluppo corrispondente all'altra nella schiera associata, e solo a tale inviluppo della schiera stessa.

Gli elementi doppi dell'involuzione sono le due quadriche di $[f]$, ciascuna delle quali è coniugata come luogo ad $n$ quadriche del fascio stesso segate da ogni $S_{n-1}$ tangente comune in $n$ coni ad invariante n-lineare nullo. Se peró $\dot{e} \Delta_{\lambda}^{2} \equiv$ o (cioè se ogni quadrica di $[f]$ gode della proprietà ora detta) ogni quadrica di $[f]$ è coniugata a tutti gli inviluppi (8).

Sulla schiera associata tornerò in seguito $\left[\mathrm{v} . \mathrm{n}^{\circ} 20\right]$.

14. Fra gli inviluppi $\left(\Phi(\beta)^{n}=0\right.$ meritano particolare menzione quelli del tipo:

$$
\beta_{\mu}^{n-k} \beta_{\nu}^{k}=0 \text {. }
$$

L'inviluppo (9), come risulta in modo semplice, è quello degli $S_{n-1}$ secanti $f_{v}$ in una quadrica b-armonica alla sezione di $f_{\mu}$.

Tenuta fissa $f_{\mu}$, quando $f_{\nu}$ descrive il fascio, l'inviluppo (9) descrive un sistema $\infty^{\mathrm{I}}$ razionale e riferito projettivamente al fascio stesso. Esistono $b$ inviluppi del sistema contenenti un $S_{n-1}$ generico. Il sistema stesso appartiene a quello lineare, ed in generale $\infty^{b}$, rappresentato dall'equazione:

$$
\beta_{\mu}^{n-b}(W \beta)^{b}=0
$$

dove $W_{\lambda}^{b}$ è una forma parametrica. Il significato di (Io) scende immediatamente da quello generale dell'inviluppo $(\Phi \beta)^{n}=0$.

La varietà di iperpiani base del sistema (10), quindi comune agli inviluppi (9), è rappresentata da:

$$
\beta_{\mu}^{n-b} \beta_{\lambda}^{h} \equiv o \quad[\text { id. risp. a }(\lambda)]
$$

cioè è costituita dagli iperpiani secanti $[f]$ in un fascio nel quale la sezione di $f_{\mu}$ conta $h+\mathrm{I}$ volte nel gruppo dei coni.

Il significato più espressivo del sistema (Io) e della varietà (I I) risulta però dalle considerazioni seguenti. Date $b+$ I quadriche di $[f]$, e cioè :

$$
f_{\xi}, f_{n}, \ldots f_{p} \text {, }
$$

esse, considerate come inviluppi, determinano il sistema, in generale $\infty^{b}$, rappresentato da :

$$
d_{1} \beta_{\xi}^{n}+d_{2} \beta_{n}^{n}+\cdots+d_{h+1} \beta_{p}^{n}=0
$$

ove le $d_{i}$ sono parametri. Il sistema (I2) ha per base la varietà:

$$
\beta_{\xi}^{n}=0, \quad \beta_{n}^{n}=0, \ldots \beta_{\rho}^{n}=0 \text {. }
$$

Se si suppone che $f_{\varepsilon}, f_{n}, \ldots f_{\rho}$ vengano successivamente a coincidere con $f_{\mu}$, le $b+$ I radici assegnate dalla (I3) a $\beta_{\lambda}^{n}$ si fondono nella radice $(b+\mathrm{I})$-pla $(\mu)$; onde la varieta (13) si riduce a (II) e quindi il sistema (I2) a (IO). Concludendo:

Il sistema (1о) è la posizione limite del sistema lineare di inviluppi individuato da 
$b+\mathrm{I}$ quadriche di $[f]$ considerate come tali, quando queste vengono successivamente a ionfondersi $\operatorname{con} f_{\mu}^{*}$ ).

La (II) è la posizione limite della varietà degli iperpiani tangenti comuni ad $b+\mathbf{I}$ quadriche di $[f]$, quando queste vengono successivamente a confondersi con $f_{\mu}$.

Se la (II) si dice varietà caratteristica d'indice $h$ relativa ad $f_{\mu}$, si puó osservare che la varietà caratteristica d'indice $b+\mathrm{I}$ relativa ad $f_{\mu} \dot{e}$ la posizione limite della varietà degli $S_{n-1}$ giacenti nella varietà caratteristica d'indice b e tangenti ad $f_{\rho}$, quando $f_{\rho}$ tende a confondersi con $f_{\mu}$. Inoltre l'inviluppo (9) si puó ritenere individuato dal contenere la varietà caratteristica d'indice $b$ relativa ad $f_{\mu}$ e quella d'indice $n-b$ relativa ad $f_{v}$.

Per $b=\mathrm{I}$ il sistema (12) si riduce alla schiera:

$$
d_{1} \beta_{\xi}^{n}+d_{2} \rho_{n}^{n}=0
$$

polare reciproca di $[f]$ rispetto a ciascuna delle quadriche rispetto alle quali sono polari reciproche $f_{\varepsilon}$ ed $f_{n}{ }^{* *}$ ). Quando $f_{\xi}$ ed $f_{n}$ vengono successivamente a confondersi con $f_{\mu}$, anche una delle quadriche rispetto a cui $f_{\xi}$ ed $f_{n}$ sono polari reciproche viene a coincidere con $f_{\mu}$, onde si giunge al notevole risultato:

La schiera $\beta_{\mu}^{n-1} \beta_{\lambda}=0$ è polare-reciproca di $[f]$ rispetto ad $f_{\mu}$.

L'inviluppo $\beta_{\mu}^{n-1} \beta_{\lambda}=0$ non è però in generale polare-reciproco di $f_{\lambda}$, come avrò occasione di dimostrare (v. $\mathrm{n}^{\circ} 2 \mathrm{I}$ ).

Dall'enunciato precedente si deduce: La varietà caratteristica d'indice I relativa ad $f_{\mu} \dot{e}$ costituita dagli $S_{n-1}$ tangenti ad $f_{\mu}$ nei punti della quartica base di $[f]$; il che si poteva prevedere anche geometricamente.

\section{$\int 4$. I complessi quadratici $(\Pi \theta)^{k}=0$.}

15. Alcuni degli sviluppi del $§$ precedente sono suscettibili di estensione quando al combinante elementare $\psi_{\lambda}^{n}$ si sostituisca il combinante elementare generico $\theta_{\lambda}^{k}$ ( $\mathrm{n}^{\mathrm{o}}$ 1o). Ed invero dal $\mathrm{n}^{\circ} 7$ (o dal $\mathrm{n}^{\circ} 8$ ) risulta intanto: Gli $S_{k-1}$ secanti $k$ quadriche $f_{\mu}, f_{v}, \ldots f_{\rho}$ $d i[f]$ in $k$ quadriche ad invariante k-lineare nullo costituiscono il complesso quadratico di equazione:

(I)

$$
\theta_{\mu} \theta_{\nu} \ldots \theta_{\rho}=0 .
$$

La (I), per il teorema del $\mathrm{n}^{\circ} 6$, si può scrivere anche sotto la forma:

(3)

$$
\left(a_{\mu} a_{y} \ldots a_{\rho} u^{\prime} u^{\prime \prime} \ldots u^{(n-k+\mathrm{x})}\right)^{2}=0 .
$$

D'altra parte, posto:

$$
\Pi_{\lambda}^{k}=(\mu \lambda)(\nu \lambda) \ldots(\rho \lambda)
$$

*) Se, come al no i2, si rappresentano gli inviluppi coi punti di uno spazio, quindi le quadriche di $[f]$, come inviluppo, coi punti di una curva, il sistema (10) è in generale rappresentato dall' $S_{b}$ osculatore alla curva nel punto corrispondente ad $f_{\mu}$.

**) Due quadriche di $S_{n}$ in generale sono polari reciproche rispetto a $2^{n}$ quadriche. Cfr. Des. Pezzo, Sulle quadriche ad $n$ - I dimensioni polari reciproche di sè stesse rispetto ad un'altra [Rend. Acc. delle Scienze Fis. e mat., Napoli, vol. XXIV, (1885), pag. I86], $\$$ III. 
la stessa (I) assume l'aspetto:

(4)

$$
(\mathrm{II} \theta)^{k}=0 .
$$

Con procedimento analogo a quello del $\mathrm{n}^{0}$ I I, si trova che, considerando $\Pi_{\lambda}^{k}$ come forma parametrica, il complesso (4) descrive un sistema lineare, il quale è $\infty^{k}$ se $[f]$ non possiede quadriche specializzate piu di $n-k+\mathrm{I}$ volte. Più in generale:

Il sistema (II $\theta)^{k}=\mathrm{o}$ di complessi quadratici è completamente indeterminato quando, $e$ solo quando, tutte le quadriche di $[f]$ sono almeno $n-k+2$ volte specializzate. In ogni altro caso, se le $r \supseteqq$ o quadriche di $[f]$ specializzate piu di $n-k+\mathrm{I}$ volte sono secate da un $S_{k-1}$ generico in quadriche da contarsi $t_{1}, t_{2}, \ldots t_{r}$ volte nel gruppo dei coni del fascio sezione, il sistema (II $\mathrm{G})^{k}=0$ è $\infty^{k-\Sigma_{t_{i}}}$, salvo l'indeterminazione del complesso per le forme $\mathbf{I}_{\lambda}^{k}$ a cui è apolare il gruppo delle quadriche specializzate piu di $n-k+\mathrm{I}$ volte, contate rispettivamente $t_{1}, t_{2}, \ldots t_{r}$ volte.

Se ne deduce in [f] l'esistenza di $\infty^{\Sigma_{i-1}}$ gruppi di $k$ quadriche secate da ogni $S_{k-1}$ in $k$ quadriche ad invariante $k$-lineare nullo.

Se poi si tiene presente che $\Pi_{\lambda}^{k}$ si puó esprimere come combinazione lineare di potenze $k$-esime di forme lineari, si può enunciare il teorema: Il sistema $(\text { II } \theta)^{k}=0$ di complessi quadratici è il sistema lineare al quale "appartiene" quello algebrico delle quadriche di $[f]$ considerate come complesso degli $S_{k-1}$ tangenti, escluse le quadriche indeterminate come tali. Se dunque i complessi quadratici di spazi $S_{k-1}$ si rappresentano coi punti di uno spazio $R$, le quadriche di $[f]$, nel senso precedente, sono rappresentate da punti di una curva d'ordine $k-\Sigma t_{i}$ (razionale) appartenente ad uno spazio $R^{\prime}$ a $k-\Sigma t_{i}$ dimensioni.

16. Suppongo che alle $r$ quadriche di $[f]$ specializzate più di $n-k+$ I volte corrispondano $i$ fattori lineari $q_{\lambda}^{\prime}, q_{\lambda}^{\prime \prime}, \ldots q_{\lambda}^{(r)}$ ed introduco la forma:

$$
\mathbf{\Omega}=\mathbf{\Omega}_{\lambda}^{\Sigma_{t_{i}}}=q_{\lambda}^{t_{1}} q_{\lambda}^{\prime \prime t_{2}} \ldots q_{\lambda}^{(r) t_{r}}
$$

analoga alla forma $\Psi$ del $n^{o}$ iा. Si ha che ad un gruppo $\Pi_{\lambda}^{k}=0$, non appartenente alla $g_{k}^{\Sigma_{i}-1}$ dei gruppi pei quali è apolare $\Omega$, corrisponde un complesso ben determinato $(\text { II } \theta)^{k}=0$; ad un complesso $(\text { II } \theta)^{k}=0$, invece, insieme al gruppo $\Pi_{2}^{k}=0$ corrispondono tutti i gruppi della $g_{k}^{\Sigma t_{i}}$ individuata dalla $g_{k}^{\Sigma t_{i}-1}$ singolare e dal gruppo stesso.

Si deduce che ai gruppi di una $g_{k}^{d}$ corrispondono $i$ complessi di un sistema lineare $\infty^{d}$ o di un sistema $\infty^{d-p-1}$, secondo che $g_{k}^{d}$ non ha in comune colla $g_{k}^{\Sigma t_{i-1}}$ singolare alcun gruppo oppure ba in comune con essa $i$ gruppi di una $g_{k}^{p}$. Etc. etc.

La relazione:

$$
(\alpha \Pi)^{k} \alpha_{\lambda}^{n-k+1} \equiv 0
$$

equivale ad $n-k+2$ equazioni lineari nei coefficienti di $\Pi_{\lambda}^{k}$, onde risulta: Se è $2 k \geqslant n+2$ e se la forma $\alpha_{\lambda}^{n+1} \dot{e}$ generale nel suo ordine, esistono $\infty^{2 k-n-2}$ forme $\Pi_{2}^{k}$ apolari ad $\alpha_{\lambda}^{n+1}$.

I gruppi corrispondenti formano una $g_{k}^{2 k-n-2}$ alla quale è subordinata la $g_{k}^{\Sigma_{l_{i-1}}}$ singolare. Per questo osservo che, se la sezione di $f_{\mu}$ con un $S_{k-1}$ generico è da contarsi $t$ volte nel gruppo dei coni del fascio sezione, si ha:

$$
\left.\theta_{\mu}^{k-t+1} \theta_{\lambda}^{t-1} \equiv 0 \quad \text { [id. risp. a }(\lambda)\right]
$$


ossia (vedi $\left.\mathrm{n}^{\circ} 6\right)$ :

$$
\left(a_{\mu}^{\prime} a_{\mu}^{\prime \prime} \ldots a_{\mu}^{(k-t+\mathrm{II}} a_{\lambda}^{\prime} a_{\%}^{\prime \prime} \ldots a_{\lambda}^{(t-5)} u^{\prime} u^{\prime \prime} \ldots u^{(n-k+\mathrm{s})}\right)^{2} \equiv \mathrm{o}
$$

qualunque siano le $u_{j}^{\prime}, u_{j}^{\prime \prime}, \ldots u_{j}^{(n-k+1)}$. E quindi lecito sostituire alle serie di variabili contragredienti altrettante serie di simboli $a_{\lambda}$ e scrivere:

ossia (vedi $\left.\mathrm{n}^{\circ} 6\right)$ :

$$
\left(a_{\mu}^{\prime} a_{\mu}^{\prime \prime} \ldots a_{\mu}^{(k-t+1)} a_{\lambda}^{\prime} a_{\lambda}^{\prime \prime} \ldots a_{\lambda}^{(n-k+t)}\right) \equiv 0
$$

$$
\alpha_{\mu}^{k-1+1} \alpha_{\lambda}^{n-k+1} \equiv 0
$$

onde segue che $f_{\mu}$ è da contarsi almeno $n-k+t+$ I volte nel gruppo dei coni di $[f]$. Si deduce cosi che $\alpha_{\lambda}^{n+1}$ contiene come fattore:

$$
\Omega^{*}=q_{\lambda}^{\prime n-k+t_{1}+1} q_{\lambda}^{\prime \prime n-k+t_{2}+1} \ldots q_{\lambda}^{(r)^{n-k+t_{r}+1}}
$$

e che perciò la spinta $(x \Pi)^{k} \alpha_{\lambda}^{n-k+1}$ possiede il fattore simbolico $(\boldsymbol{\Omega} I)^{\Sigma t_{i}}$; il che dimostra, come si voleva, che, se è $\Omega$ apolare a $\mathbf{\Pi}_{\lambda}^{k}\left[\right.$ cioè $\left.(\Omega \mathbf{I I})^{\Sigma_{t_{i}}} \mathbf{I}_{\lambda}^{k-\Sigma t_{i}} \equiv 0\right]$, è pure $\mathbf{I}_{\lambda}^{k}$ apolare ad $\alpha_{\lambda}^{n+1}\left[\right.$ cioè $\left.(\alpha \Pi)^{k} \alpha_{\lambda}^{n-k+1} \equiv 0\right]$.

Applicando il criterio generale sopra esposto si puó concludere:

Se $\dot{e} 2 k \gg n+2+\Sigma t_{i}$, alla $g_{k}^{2 k-n-2}$ dei gruppi apolari a quello dei coni corrisponde un sistema lineare $2 k-n-2-\Sigma t_{i}$ volte infinito. $\mathrm{Si}$ intende che il risultato vale solo in generale intendendosi esclusi $i$ casi in cui la dimensione della serie in questione superi $2 k-n-2$.

Per stabilire un significato geometrico del sistema di complessi ora considerato si ricordi $\left(n^{\circ} 6\right)$ che, ponendo, come sopra, $\Pi_{\lambda}^{k}=(\mu \lambda)(v \lambda) \ldots(p \lambda)$, la

si scrive pure:

$$
(\alpha \Pi)^{k} \alpha_{\lambda}^{n-k+1}=0
$$

$$
\left(a_{\mu} a_{\nu} \ldots a_{\rho} a_{\lambda}^{\prime} a_{\lambda}^{\prime \prime} \ldots a_{\lambda}^{(n-k+3)}\right)^{2}=0
$$

Dal confronto delle $(5)$ e $(6)$, tenuta presente una definizione data $a l n^{\circ} 5$ si deduce : Il complesso $(\Pi \theta)^{k}=0 \dot{e}$ in generale armonico ad $n-k+\mathrm{I}$ quadriche di $[f]$ $i$ cui parametri sono forniti dall'equazione $(\alpha \mathrm{II})^{k} \alpha_{\lambda}^{n-k+1}=0$. I complessi $(\mathrm{II} \theta)^{k}=0$ corrispondenti alle forme $\Pi_{\lambda}^{k}$ apolari ad $\alpha_{\lambda}^{n+1}$, ed essi soli, sono armonici a tutte le quadriche di $[f]$.

In generale, e sempre nell'ipotesi $2 k \gg n+2$, alla $g_{k}^{2 k-n-2}$ dei gruppi apolari ad $\alpha_{\lambda}^{n+1}$ nel campo binario è coniugata la $g_{k}^{n-k+1}$ dei gruppi polari ad $\alpha_{\lambda}^{n+1}$ in senso esteso, cioè dei gruppi :

(7)

$$
(\alpha V)^{n-k+1} \alpha_{\lambda}^{k}=0
$$

essendo $V_{\lambda}^{n-k+1}$ una forma parametrica. Il complesso:

$$
(\alpha V)^{n-k+1}(\alpha \theta)^{k}=0
$$

corrispondente al gruppo (7) può essere definito come complesso degli $S_{k-1}$ tangenti a $k$ quadriche costituenti insieme a quelle fornite $d a V_{\lambda}^{n-k+1}=0$ un gruppo ad invariante $(n+1)$-lineare nullo. In generale il sistema (8) di complessi è $\infty^{n-k+1}$ e si puó ritenere un'estensione della schiera associata (cfr. $n^{\circ}$ I 3 ).

Caso particolare di (8) è il complesso:

$$
\alpha_{\sigma}^{n-k+1}(\alpha \theta)^{k}=0
$$


degli $S_{k-1}$ secanti in $k$ quadriche ad invariante k-lineare nullo le $k$ quadriche di $[f]$ $k$-armoniche ad $f_{\sigma}$.

I7. Il presente $\mathrm{n}^{\circ}$ contiene l'estensione di alcuni fra $\mathrm{i}$ risultati del $\mathrm{n}^{\circ}$ I4. Il complesso:

(9)

$$
\theta_{\mu}^{k-b} \theta_{\nu}^{h}=0
$$

degli $S_{k-1}$ secanti $f_{\nu}$ in una quadrica b-armonica alla sezione di $f_{\mu}$, al variare di $f_{\nu}$, descrive un sistema $\infty^{\mathrm{I}}$ razionale.

Esistono $b$ complessi del sistema contenenti un $S_{k-1}$ generico ed il sistema stesso appartiene a quello lineare, e in generale $\infty^{b}$, rappresentato $\mathrm{da}$

La base di (Io) è la:

$$
\theta_{\mu}^{k-b}(W \theta)^{h}=0 .
$$

$$
\left.\theta_{\mu}^{k-h} \theta_{\lambda}^{h} \equiv o \quad \text { [id. risp. a }(\lambda)\right]
$$

degli $S_{k-1}$ secanti $[f]$ in un fascio nel quale la sezione di $f_{\mu}$ conta $b+\mathrm{I}$ volte nel gruppo dei coni.

Il sistema ( 10 ) è la posizione limite del sistema lineare di complessi quadratici individuato da $b+$ I quadriche di $[f]$ pensate come complesso dei loro $S_{k-1}$ tangenti, quando le $b+$ I quadriche vengono successivamente a coincidere con $f_{\mu}$. La varietà (I I) è la posizione limite di quella degli $S_{k-1}$ tangenti ad $b+$ I quadriche di $[f]$ quando queste vengono a coincidere successivamente con $f_{\mu}$. Etc. etc.

\section{I fasci dotati di piramide polare.}

I8. Nel presente $\int$ viene considerato il caso in cui esiste almeno una piramide di $n+\mathrm{I}$ vertici polare (autoconjugata) rispetto a tutte le quadriche di $[f]$, ossia il caso in cui gli esponenti dei divisori elementari di WeIERstrass sono tutti uguali all'unità, se il fascio non è di quadriche specializzate od il caso in cui sono tali gli esponenti dei divisori elementari relativi al fascio sezione con un $S_{n-k}$ generico. se la quadrica corrente di $[f]$ è $k$ volte specializzata. Assunta la piramide polare come fondamentale, l'equazione della quadrica $f_{\lambda}$ di $[f]$ prende l'aspetto :

$$
e_{\lambda}^{\prime} x_{1}^{2}+e_{\lambda}^{\prime \prime} x_{2}^{2}+\cdots+e_{\lambda}^{(n+1)} x_{n+1}^{2}=0 \text {, }
$$

ove le $e_{\lambda}^{(i)}$ sono forme lineari. Se di queste nessuna è identicamente nulla, la quadrica corrente di $[f]$ non è specializzata, e ad un fattore lineare nel quale coincidano $r \gg \mathbf{I}$ delle $e_{\lambda}^{(i)}$ (a meno di coefficienti numerici), corrisponde una quadrica $r$ volte specializzata. Se invece $k$ delle $e_{\lambda}^{(i)}$ sono identicamente nulle, la quadrica corrente di $[f]$ è $k$-volte specializzata, e ad un fattore lineare nel quale coincidano $r \gg \mathrm{I}$ delle rimanenti corrisponde una quadrica $r+k$ volte specializzata. In ogni caso risulta facilmente:

Se inoltre si pone:

$$
x_{\lambda}^{n+1}=e_{\lambda}^{\prime} e_{\lambda}^{\prime \prime} \ldots e_{\lambda}^{(n+1)} \text {. }
$$

$$
E_{\lambda}^{(i)^{n}}=e_{\lambda}^{\prime} e_{\lambda}^{\prime \prime} \ldots e_{\lambda}^{(i-1)} e_{\lambda}^{(i+1)} \ldots e_{\lambda}^{(n+1)}
$$


si deduce che l'equazione tangenziale della quadrica (I) è :

$$
E_{\lambda}^{\prime n} u_{1}^{2}+E_{\lambda}^{\prime \prime n} u_{2}^{2}+\cdots+E_{\lambda}^{(n+1)^{n}} u_{n+1}^{2}=0,
$$

indeterminata per ogni $f_{2}$, quando, e solo quando, almeno due delle $e_{\lambda}^{(i)}$ siano identicamente nulle, in accordo con quanto precede.

Poichè (4) coincide con $\beta_{2}^{n}=0$, si ha the più in generale l'inviluppo $(\Phi \beta)^{n}=0$ del $\ 3$ è rappresentato da :

$$
\left(\Phi E^{\prime}\right)^{n} u_{\mathrm{r}}^{2}+\left(\Phi E^{\prime \prime}\right)^{n} u_{2}^{2}+\cdots+\left(\Phi E^{(n+\mathrm{r})}\right)^{n} u_{n+\mathrm{r}}^{2}=0
$$

e su di questa equazione si possono facilmente ritrovare per il caso presente $i$ risultati stabiliti, per $[f]$ qualunque, al $n^{\circ}$ I I sulla dimensione del sistema $(\Phi \beta)^{n}=0$.

Ogni piramide polare rispetto a tutte le quadriche di $[f]$ è pure polare rispetto ad ogni inviluppo (5), come si deduce immediatamente dal teorema dato in principio del $\mathrm{n}^{\mathrm{o}}$ 12. Ma, tenuto conto caso per caso della dimensione del sistema ( 5 ), risulta che reciprocamente ogni inviluppo dotato di tale proprietà appartiene al sistema. Limitando l'osservazione al caso di un fascio generale, cioè [ [ I I ... I], si ha: Per un fascio generale di quadriche, ogni quadrica rispetto alla quale è polare la piramide dei vertici dei coni è inviluppo degli iperpiani secanti $n$ quadriche ben determinate del fascio in $n$ quadriche ad invariante $n$-lineare nullo. La forma $\Phi_{2}^{n}$ corrispondente alla quadrica di equazione tangenziale :

$$
p_{1} u_{1}^{2}+p_{2} u_{2}^{2}+\cdots+p_{n+1} u_{n+1}^{2}=0
$$

è individuata come coniugata alle $n$ forme che si ottengono dai minori della matrice

$$
\left\|\begin{array}{cccc}
E_{\lambda}^{\prime n} & E_{\lambda}^{\prime \prime n} & \cdots & E_{\lambda}^{(n+1) n} \\
p_{\mathrm{r}} & p_{2} & \cdots & p_{n+1}
\end{array}\right\|
$$

tenendo fissa una colonna assegnata, per es. la prima; e puó essere posta sotto l'aspetto:

$$
\Phi_{\lambda}^{n}=\frac{p_{1}}{\left(E^{\prime} e^{\prime}\right)^{n}} e_{\lambda}^{\prime n}+\frac{p_{2}}{\left(E^{\prime \prime} e^{\prime \prime}\right)^{n}} e_{\lambda}^{\prime \prime n}+\cdots+\frac{p_{n+1}}{\left(E^{(n+1)} e^{(n+1)}\right)^{n}} e_{\lambda}^{(n+1) n} .
$$

19. D'ora innanzi suppongo $\alpha_{\lambda}^{n+1} \neq 0$. Risulta allora semplicemente che gli inviluppi $(\Phi \beta)^{n}=0$ specializzati corrispondono alle forme $\Phi_{\lambda}^{n}$ coniugate ad una almeno delle $E_{\lambda}^{(i)^{n}}$. Più precisamente, se il fascio è [ I I I $\ldots$ I] ], ad una $\boldsymbol{\Phi}_{\lambda}^{n}$ coniugata ad esempio ad $E_{\lambda}^{\prime n}, E_{\lambda}^{\prime \prime n} \ldots E_{\lambda}^{(j)^{n}}$ corrisponde un inviluppo $j$ volte specializzato ed avente per nucleo una quadrica dell' $S_{n-i}$ :

$$
x_{1}=0, \quad x_{2}=0, \ldots x_{j}=0 .
$$

Dato un gruppo $M_{\lambda}^{n+1}=0$, si considerino i suoi gruppi primi polari $M_{s} M_{\lambda}^{n}=0$, costituenti in generale una $g_{n}^{\prime}$ nel campo binario del fascio. Alla $g_{n}^{\prime}$ corrisponde in generale $\left(n^{0}\right.$ I 3$)$ la schiera:

$$
M_{\sigma}(M \beta)^{n}=\mathrm{o}
$$

riferita projettivamente ad $[f]$, corrispondendosi $(7)$ ed $f_{\sigma}$. La (7) per il numero precedente si puó scrivere:

$$
M_{\sigma}\left(M E^{\prime}\right)^{n} u_{\mathrm{r}}^{2}+M_{\sigma}\left(M E^{\prime \prime}\right)^{n} u_{2}^{2}+\cdots+M_{\sigma}\left(M E^{(n+1)}\right)^{n} u_{n+\mathrm{I}}^{2}=0 .
$$

Rend. Circ. Matem. Palermo, t. XXIII (1907). - Stampato il 4 aprile 1907. 
Si supponga ora che il gruppo $M_{i}^{n+1}=0$ sia conjugato a quello dei coni, o, ciò che è lo stesso (vedi $\mathrm{n}^{\circ} 7$ ), sia un gruppo di quadriche ad invariante $(n+\mathrm{I}$ )-lineare nullo. In tale ipotesi è :

(9)

$$
(x M)^{n+1}=0 \text {, }
$$

condizione questa necessaria e sufficiente perchè le forme lineari $M_{\sigma}\left(M E^{(i)}\right)^{n}$ differiscano rispettivamente dalle $e_{\sigma}^{(i)}$ solo per fattori costanti, ossia perchè nella projettività posta tra $[f]$ e $(7)$ ai coni di $[f]$ corrispondano ordinatamente gli inviluppi specializzati di (7). Si ha cosi :

$$
\left\{\begin{array}{l}
M_{\sigma}\left(M E^{\prime}\right)^{n}=p_{1} e_{\sigma}^{\prime} \\
M_{\sigma}\left(M E^{\prime \prime}\right)^{n}=p_{2} e_{\sigma}^{\prime \prime} \\
\ldots \ldots \ldots \ldots \ldots \\
M_{\sigma}\left(M E^{(n+\mathrm{I})}\right)^{n}=p_{n+\mathrm{I}} e_{\sigma}^{(n+\mathrm{I})}
\end{array}\right.
$$

dove le $\rho_{i}$ sono appunto fattori costanti. Se ne deduce, in modo semplice, che la quadrica $f_{\sigma}$ e l'inviluppo (7) sono polari-reciproci rispetto a ciascuna delle $2^{n}$ quadricheinviluppo che si ottengono dalla:

$$
\sqrt{\rho_{1}} u_{1}^{2} \pm \sqrt{\rho_{2}} u_{2}^{2} \pm \cdots \pm \sqrt{\rho_{n+1}} u_{n+1}^{2}=0
$$

fissandone $i$ doppi segni in tutti $i$ modi possibili ${ }^{*}$ ).

Se $[f]$ è di tipo [ [ I I I ... I] risulta immediatamente che gli inviluppi (I I) appartengono al sistema $(\Phi \beta)^{n}=0$; anzi le corrispondenti forme $\Phi_{\lambda}^{n}$ sono assegnate dalla (6). Suppongo ora che $[f]$ sia di tipo $[($ II I ... I ) I I ... I] e più precisamente che le $e_{\lambda}^{\prime}, e_{\lambda}^{\prime \prime}, \ldots e_{\lambda}^{(r)}$ coincidano a meno di fattori numerici. In tal caso è lecito porre:

$$
e_{\lambda}^{\prime}: e_{\lambda}^{\prime \prime}: \cdots: e_{\lambda}^{(r)}=b_{1}: b_{2}: \cdots: b_{r},
$$

essendo $b_{1}, b_{2}, \ldots b_{r}$ costanti non nulle, da cui si deduce:

$$
\left(\Phi E^{\prime}\right)^{n}:\left(\Phi E^{\prime \prime}\right)^{n}: \cdots:\left(\Phi E^{(r)}\right)^{n}=\frac{\mathrm{I}}{b_{1}}: \frac{\mathrm{I}}{h_{2}}: \cdots: \frac{\mathrm{I}}{b_{r}} .
$$

Segue che, se l'inviluppo

$$
p_{\mathrm{r}} u_{\mathrm{r}}^{2}+p_{2} u_{2}^{2}+\cdots+p_{n+1} u_{n+1}^{2}=0
$$

appartiene a $(\Phi \beta)^{n}=0$, si ha :

$$
p_{1}: p_{2}: \cdots: p_{r}=\frac{\mathrm{I}}{h_{1}}: \frac{\mathrm{I}}{h_{2}}: \cdots: \frac{\mathrm{I}}{h_{r}} ;
$$

ma sussiste anche la proposizione reciproca, come risulta quando si tenga presente la dimensione del sistema. D'altra parte, dalle (IO), (I 2), (I3) si ricava:

$$
p_{1}: p_{2}: \cdots: p_{r}=\left(\frac{I}{h_{1}}\right)^{2}:\left(\frac{I}{h_{2}}\right)^{2}: \cdots:\left(\frac{I}{b_{r}}\right)^{2},
$$

onde è possibile determinare $r$ - I numeri $n_{2}, n_{3}, \ldots n_{r}$, ciascuno dei quali è uguale

*) S'intende che con $\sqrt{p_{i}}$ è qui indicata una delle due radici quadrate di $p_{i}$. Le quadriche (II) formano un gruppo del tipo ricordato al $\mathrm{n}^{\circ} \mathrm{I4}$ in nota. 
ad I oppure a $-\mathrm{I}$, in modo che sia:

$$
\sqrt{\rho_{1}}: n_{2} \sqrt{\rho_{2}}: \cdots: n_{1} \sqrt{\rho_{r}}=\frac{1}{b_{1}}: \frac{1}{b_{2}}: \cdots: \frac{1}{b_{r}} .
$$

Se ne deduce che, nel caso presente, fra gli inviluppi (I I) $i 2^{n-r+1}$ forniti dalle (18) $\sqrt{\rho_{1}} u_{1}^{2}+n_{2} \sqrt{\rho_{2}} u_{2}^{2}+\cdots+n_{r} \sqrt{\rho_{r}} u_{r}^{2} \pm \sqrt{\rho_{r+1}} u_{r+1}^{2} \pm \cdots \pm \sqrt{\rho_{n+1}} u_{n+1}^{2}=0$, ed essi soli, appartengono al sistema $\left(\Phi(\xi)^{n}=0\right.$. L'estensione del risultato a ciascuno dei tipi considerati nel presente $\mathrm{n}^{\circ}$ è ora assai semplice e conduce all'enunciato:

Se [f] possiede $l$ quadriche specializzate distinte ed $\dot{e}(\alpha M)^{n+1}=0$, la schiera $M_{\sigma}(M \beta)^{n}=$ o è polare-reciproca di $[f]$ rispetto a $2^{l-1}$ quadriche-inviluppo del sistema $(\Phi \beta)^{n}=0$, corrispondendosi nella polarità la quadrica $f_{\sigma}$ e l'inviluppo $M_{\sigma}\left(M(\beta)^{n}=0\right.$.

E però specialmente degno di nota che, inversamente, la scbiera polare-reciproca di [f] rispetto ad una quadrica-inviluppo del sistema $\left(\boldsymbol{\Phi}(\dot{\beta})^{n}=0\right.$ è sempre costituita dagli inviluppi $\left(\Phi()^{n}=\right.$ o corrispondenti ai gruppi primi polari di un gruppo $M_{\lambda}^{n+1}=0$ coniugato a quello dei coni.

Si consideri infatti la schiera polare di $[f]$ rispetto alla quadrica-inviluppo:

$$
\pi_{1} u_{1}^{2}+\pi_{2} u_{2}^{2}+\cdots+\pi_{n+1} u_{n+1}^{2}=0
$$

del sistema $(\Phi \beta)^{n}=0$. La schiera stessa è rappresentata da :

$$
\pi_{1}^{2} e_{\lambda}^{\prime} u_{1}^{2}+\pi_{2}^{2} e_{\lambda}^{\prime \prime} u_{2}^{2}+\cdots+\pi_{n+1}^{2} e_{\lambda}^{(n+1)} u_{n+1}^{2}=0 .
$$

Se $[f]$ è di tipo [I I I ... I I], le $\pi_{i}$ sono arbitrarie ed una $M_{\lambda}^{n+1}$ coniugata ad $x_{\lambda}^{n+1}$, del resto qualunque, è fornita da:

$$
M_{\lambda}^{n+1} \equiv \delta_{1} e_{\lambda}^{\prime n+1}+\delta_{2} e_{\lambda}^{\prime \prime n+1}+\cdots+\delta_{n+1} e_{\lambda}^{(n+1)^{n+1}}
$$

ove le $\delta_{i}$ sono pure arbitrarie. Ne segue:

$$
M_{\sigma}\left(M E^{(i)}\right)^{n}=\delta_{i}\left(e^{(i)} E^{(i)}\right)^{n} e_{\sigma}^{(i)},
$$

onde perchè (20) coincida con (8) basta porre in (2I):

$$
\delta_{i}=\frac{\pi_{i}^{2}}{\left(e^{(i)} E^{(i)}\right)^{n}}
$$

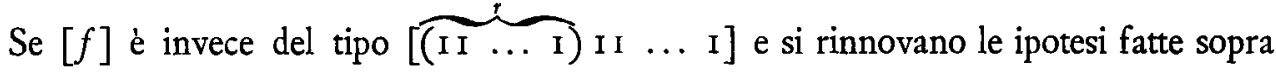
per questo caso, in virtù delle (I5) si ha:

$$
\pi_{\mathrm{r}}: \pi_{2}: \cdots: \pi_{r}=\frac{\mathrm{I}}{h_{\mathrm{I}}}: \frac{\mathrm{I}}{h_{2}}: \cdots: \frac{\mathrm{I}}{h_{r}}
$$

ed ogni forma $M_{\lambda}^{n+1}$ coniugata ad $\alpha_{\lambda}^{n+1}$ è del tipo:

(24) $M_{\lambda}^{n+1}=\delta_{\lambda}^{r-1} e_{\lambda}^{n-r+2}+\delta_{r+1} e_{\lambda}^{(r+1)^{n+1}}+\delta_{r+2} e_{\lambda}^{(r+2)^{n+1}}+\cdots+\delta_{n+1} e_{\lambda}^{(n+1)^{n+1}}$, ove $\delta_{\lambda}^{r-1}$ è una forma parametrica, le $\delta_{r+1}, \delta_{r+2}, \ldots \delta_{n+1}$ sono costanti arbitrarie, $e_{2}$ differisce per un fattore costante da ciascuna delle $e_{\lambda}^{\prime}, e_{\lambda}^{\prime \prime}, \ldots e_{\lambda}^{(r)}$. Se si tiene presente che $e_{\lambda}$ è fattore $(r-\mathrm{I})$-plo oppure $r$-plo per $E_{\lambda}^{(i)^{n}}$ secondo che è $i \leq r$ oppure $i>r$, si ha:

$$
\begin{aligned}
& \left(E^{(i)} e\right)^{n-r+2} E_{\lambda}^{(i)^{r-2}} \equiv 0, \text { per } i \leq r, \\
& \left(E^{(i)} e\right)^{n-r+1} E_{\lambda}^{(i)^{r-1}} \equiv 0, \text { per } i>r
\end{aligned}
$$


onde facilmente segue:

$$
\left\{\begin{array}{l}
M_{\sigma}\left(M E^{(i)}\right)^{n}=\frac{n-r+2}{n+1} e_{\sigma}\left(\delta E^{(i)}\right)^{r-s}\left(e E^{(i)}\right)^{n-r+1}, \text { per } i \leq r, \\
M_{\sigma}\left(M E^{(i)}\right)^{n}=\delta_{i} e_{\sigma}^{(i)}\left(e^{(i)} E^{(i)}\right)^{n}, \text { per } i>r .
\end{array}\right.
$$

Perchè (20) coincida con (8) deve essere:

$$
\left\{\begin{array}{c}
M_{\sigma}\left(M E^{\prime}\right)^{n}: \cdots: M_{\sigma}\left(M E^{(r)}\right)^{n}: M_{\sigma}\left(M E^{(r+1)}\right)^{n}: \cdots: M_{\sigma}\left(M E^{(n+\mathrm{r})}\right)^{n} \\
=\pi_{s}^{2} e_{\sigma}^{\prime}: \cdots: \pi_{r}^{2} e_{\sigma}^{(r)}: \pi_{r+1}^{2} e_{\sigma}^{(r+1)}: \cdots: \pi_{n+1}^{2} e_{\sigma}^{(n+1)} .
\end{array}\right.
$$

Ora dalle (12), (I3), (23) si deduce:

$$
M_{\sigma}\left(M E^{\prime}\right)^{n}: \cdots: M_{\sigma}\left(M E^{(r)}\right)^{n}=\pi_{\mathrm{r}}^{2} e_{\sigma}^{\prime}: \cdots: \pi_{r}^{2} e_{\sigma}^{(r)},
$$

onde, posto per semplicità ad es. $e_{\lambda}=k_{1} e_{\lambda}^{\prime}$, se in (24) si fa:

$$
\delta_{i}=k_{\mathrm{I}} \frac{n-r+2}{n+\mathrm{I}} \frac{\pi_{i}^{2}\left(\delta E^{\prime}\right)^{r-1}\left(e E^{\prime}\right)^{n-r+\mathrm{I}}}{\pi_{\mathrm{i}}^{2}\left(e^{(i)} E^{(i)}\right)^{n}} \quad(i=r+\mathrm{I}, r+2, \ldots, n+\mathrm{I})
$$

la (26) è soddisfatta. In questo caso peró la determinazione di $M_{\lambda}^{n+1}$ si puó fare in $\infty^{r-1}$ modi, rimanendo arbitraria la scelta di $\delta_{\lambda}^{r-1}$; il che è in accordo coll'osservazione in principio del $\mathrm{n}^{0} \mathrm{r} 3$.

La dimostrazione del teorema per un fascio qualunque di quadriche non specializzate e dotato di piramide polare è una generalizzazione della precedente e viene omessa per brevità.

20. Le considerazioni ora svolte possono condurre ad ulteriori proprietà per la schiera associata introdotta al $\mathrm{n}^{\circ} \mathrm{I} 3$.

Premetto un'osservazione. Se sulla schiera àssociata ad $[f]$ si opera in modo correlativo a quello seguito per ottenere da $[f]$ la schiera stessa, si viene a costruire un fascio che diro associato alla scbiera. Se avviene che il fascio associato alia schiera associata coincide col fascio dato, dirò brevemente che sussiste il teorema di reciprocità.

Ora si può dimostrare che per $n$ pari sussiste il teorema di reciprocità. Ed invero in tal caso è $\left(x \alpha^{\prime}\right)^{n+1}=0$, onde $\left(\mathrm{n}^{0}\right.$ I 9$)$ il fascio $[f]$ e la schiera associata sono polari reciproci rispetto a $2^{n}$ quadriche. Ne segue immediatamente che appunto il fascio associato alla schiera associata ad $[f]$ è lo stesso [f]; anzi ciò avviene in modo che una quadrica e un inviluppo corrispondenti nella projettivita tra $[f]$ e la schiera associata posta al $\mathrm{n}^{0} \mathrm{I} 3$, sono pure corrispondenti in quella che correlativamente si puó porre fra la schiera ed $[f]$ considerato come fascio associato a questa.

Il risultato può essere anche stabilito direttamente e completato come segue. Dalla

di Gordan *) si ha:

$$
\left(\begin{array}{ccc}
E_{\lambda}^{(i)^{n}}, & e_{\lambda}^{(i)}, & E_{\lambda}^{(i)^{n}} \\
0 & n & 0
\end{array}\right)
$$

*) Se $F_{1}, F_{2}, F_{3}$ sono tre forme binarie risp. d'ordine $n_{1}, n_{2}, n_{3}$, con $\left(\begin{array}{c}F_{1} F_{2} F_{3} \\ p_{1} p_{2} p_{3}\end{array}\right)$, dove è $p_{r} \supseteq 0$ e, per $p_{1} \neq 0$, si ha $p_{2}+p_{3}=n_{1}$, mentre, per $p_{1}=0$ si ha $p_{2}+p_{3} \leqslant n_{1}$, viene richiamata la se- 


$$
\left(E_{\lambda}^{\left(i^{n}\right.} e_{\lambda}^{(i)}, E_{\lambda}^{(i)^{n}}\right)^{n}+\frac{n}{n+\mathrm{I}}\left[\left(E_{\lambda}^{(i)^{n}}, e_{\lambda}^{(i)}\right)^{\mathrm{I}}, E_{\lambda}^{(i)^{n}}\right]^{n-1}=\left(E^{(i)} \bar{E}^{(i)}\right)^{n} e_{\lambda}^{(i)}
$$

ove si è posto $\bar{E}_{\lambda}^{(i)^{n}}=E_{\lambda}^{(i)^{n}}$; e dalla:

si ha:

$$
\left(\begin{array}{ccc}
E_{\lambda}^{(i)^{n}}, & e_{\lambda}^{(i)}, & E_{\lambda}^{(i)^{n}} \\
0 & n-\mathrm{I} & \mathrm{I}
\end{array}\right)
$$

$$
\left[\left(E_{\lambda}^{(i)^{n}}, e_{\lambda}^{(i)}\right)^{1}, E_{\lambda}^{(i)^{n}}\right]^{n-1}=\left[\left(E_{\lambda}^{(i)^{n}}, E_{\lambda}^{(i)^{n}}\right)^{n-1}, e_{\lambda}^{(i)}\right]^{\mathrm{s}}+\frac{1}{2}\left(E^{(i)} \bar{E}^{(i)}\right)^{n} e_{\lambda}^{(i)}
$$

onde si deduce:

$$
\alpha_{\lambda}\left(\alpha E^{(i)}\right)^{n}=-\frac{n}{n+\mathrm{I}}\left[\left(E_{\lambda}^{(i)^{n}}, E_{\lambda}^{(i)^{n}}\right)^{n-1}, e_{\lambda}^{(i)}\right]^{1}+\frac{n+2}{2(n+\mathrm{I})}\left(E^{(i)} \bar{E}^{(i)}\right)^{n} e_{\lambda}^{(i)}
$$

qualunque sia $n$. Ma, riprendendo l'ipotesi di $n$ pari, si ha più semplicemente:

$$
\alpha_{\lambda}\left(\alpha E^{(i)}\right)^{n}=\frac{n+2}{2(n+1)}\left(E^{(i)} \bar{E}^{(i)}\right)^{n} e_{\lambda}^{(i)}
$$

e l'equazione della schiera associata al fascio

$$
\sum_{i}^{n+1} e_{\lambda}^{(i)} x_{i}^{2}=0
$$

prende la forma:

Se pongo per brevità :

$$
\sum_{i}^{n+1}\left(E^{(i)} \bar{E}^{(i)}\right)^{n} e_{\lambda}^{(i)} u_{i}^{2}=0
$$

ed

$$
\boldsymbol{E}_{\lambda}^{(i)^{n}}=\overline{\boldsymbol{E}}_{\lambda}^{(i)^{n}} \doteq \varepsilon_{\lambda}^{\prime} \varepsilon_{\lambda}^{\prime \prime} \ldots \varepsilon_{\lambda}^{(i-1)} \varepsilon_{\lambda}^{(i+1)} \ldots \varepsilon_{\lambda}^{(n+1)},
$$

il fascio associato alla schiera (3o) è rappresentato da:

$$
\sum_{I}^{n+1}\left(\boldsymbol{E}^{(i)} \overline{\boldsymbol{E}}^{(i)}\right)^{n} \varepsilon_{\lambda}^{(i)} x_{i}^{2}=0 .
$$

Ma dalle (28), (3I), (32) si deduce:

$$
\left(\bar{E}^{(i)} \overline{\boldsymbol{E}}^{(i)}\right)^{n} \varepsilon_{\lambda}^{(i)}=\left(\frac{n+2}{2(n+\mathrm{I})}\right)^{2 n+1}\left[\left(E^{\prime} \bar{E}^{\prime}\right)^{n}\left(E^{\prime \prime} \bar{E}^{\prime \prime}\right)^{n} \ldots\left(E^{(n+1)} \bar{E}^{(n+1)}\right)^{n}\right]^{2} e_{\lambda}^{(i)},
$$

onde (33) in generale coincide con (29), cioè sussiste il teorema di reciprocità. L'unico caso di eccezione è dato dall'annullarsi di uno degli invarianti $\left(E^{(i)} \bar{E}^{(i)}\right)^{n}$, che conduce

guente formola :

$$
\begin{gathered}
\sum_{0} \frac{\left(\begin{array}{c}
n_{2}-p_{1}-p_{3} \\
k
\end{array}\right)\left(\begin{array}{c}
p_{2} \\
k
\end{array}\right)}{\left(\begin{array}{c}
n_{1}+n_{2}-2 p_{3}-k+\mathrm{I} \\
k
\end{array}\right)}\left[\left(F_{1}, F_{2}\right)^{p_{3}+k}, F_{3}\right]^{p_{1}+p_{2}-k} \\
=(-\mathrm{I})^{p_{1}} \sum_{0} \frac{\left(\begin{array}{c}
n_{3}-p_{1}-p_{2} \\
k
\end{array}\right)\left(\begin{array}{c}
p_{3} \\
k
\end{array}\right)}{\left(\begin{array}{c}
n_{1}+n_{3}-2 p_{2}-k+\mathrm{I} \\
k
\end{array}\right)}\left[\left(F_{1}, F_{3}\right)^{p_{2}+k}, F_{2}\right]^{p_{1}+p_{3}-k} .
\end{gathered}
$$

Per questa v. Gordan, Ueber das Formensystem binarer Formen (Leipzig, I875); pag. II, form. (III). 
all'indeterminazione di (33), risultato prevedibile per lo specializzarsi dell'inviluppo corrente $(30)$ della schiera associata.

La (28), tenute presenti le (IO) e (II), permette di scrivere le equazioni delle $2^{n}$ quadriche sopra ricordate, riunendole nella:

$$
\sqrt{\left(E^{\prime} \bar{E}^{\prime}\right)^{n}} u_{\mathrm{r}}^{2} \pm \sqrt{\left(E^{\prime \prime} \bar{E}^{\prime \prime}\right)^{n}} u_{2}^{2} \pm \cdots \pm \sqrt{\left(E^{(n+\mathrm{r})} \overline{\left.\bar{E}^{(n+1)}\right)^{n}}\right.} u_{n+\mathrm{r}}^{2}=0
$$

ove $\mathrm{i}$ doppi segni sono da prendersi in tutti $\mathrm{i}$ modi.

Per $n$ dispari in generale non sussiste il teorema di reciprocità, come si puó verificare ad es. per $\left.n=3^{*}\right)$. Se però il gruppo dei coni è ad invariante $(n+1)$-lineare nullo, cioè (vedi $\mathrm{n}^{0} 7$ ) se si ha: $\left(\alpha \alpha^{\prime}\right)^{n+r}=0$, sono ancora applicabili le considerazioni del $\mathrm{n}^{0} \mathrm{I}$, quindi $[f]$ e la schiera associata sono polari-reciproci rispetto a $2^{n}$ quadriche. In questo caso sussiste dunque il teorema di reciprocità, e precisamente in modo che una quadrica di $[f]$ e l'inviluppo della schiera associata corrispondentisi nella projettivita del $\mathrm{n}^{0} \mathrm{I}_{3}$ si corrispondono pure in quella posta correlativamente fra la schiera ed $[f]$, pensato come fascio associato. Un altro caso in cui, anche per $n$ dispari, sussiste il teorema di reciprocità si incontrerà fra breve (vedi $\mathrm{n}^{0}{ }^{2} 23$ ).

2I. $\mathrm{Al}^{0} \mathrm{I}_{4}$ si è osservato che la schiera:

$$
d_{1} \beta_{\xi}^{n}+d_{2} \beta_{n}^{n}=0
$$

è polare-reciproca di $[f]$ rispetto a ciascuna delle $2^{n}$ quadriche rispetto a cui sono polarireciproche $f_{\xi}$ ed $f_{n}$. Il risultato puó essere completato e messo in relazione col $\mathbf{n}^{\circ} \mathrm{Ig}$. Percio, posto:

(35)

$$
M_{\lambda}^{n+1}=\alpha_{n}^{n+1}(\xi \lambda)^{n+1}-\alpha_{\xi}^{n+1}(n \lambda)^{n+1},
$$

osservo che si ha:

(36)

onde segue:

(37)

$$
M_{\sigma} M_{\lambda}^{n}=\alpha_{n}^{n+1}(\xi \sigma)(\xi \lambda)^{n}-\alpha_{\xi}^{n+1}(n \sigma)(n \lambda)^{n},
$$

$$
M_{\sigma}(M \beta)^{n}=-\alpha_{n}^{n+1}(\xi \sigma) \beta_{\xi}^{n}+\alpha_{\xi}^{n+1}(n \sigma) \beta_{n}^{n},
$$

e (34) coincide con $M_{\sigma}(M \beta)^{n}=0$, quando vi si ponga:

$$
\begin{aligned}
& d_{1}=-\alpha_{n}^{n+1}(\zeta \sigma), \\
& d_{2}=\alpha_{\xi}^{n+1}(n \sigma) .
\end{aligned}
$$

Viene così stabilito quale inviluppo di (34) corrisponda ad $f_{\sigma}$ nella polarità di cui sopra, che, essendo $(\alpha M)^{n+1}=0$, coincide con quella definita dalle considerazioni del $\mathrm{n}^{\circ} 19$.

Tenuta presente la (36), l'equazione della schiera (34) si puó pure scrivere cosi:

ossia :

$$
\sum_{i}^{n+1}\left[\alpha_{n}^{n+1}(\xi \sigma) E_{\xi}^{(i)^{n}}-\alpha_{\xi}^{n+1}(n \sigma) E_{n}^{(i) n^{n}}\right] u_{i}^{2}=0,
$$

$$
\sum_{i}^{n+1} E_{\xi}^{(i)^{n}} E_{n}^{(i)^{n}}\left[(\xi \sigma) e_{n}^{(i)}-(n \sigma) e_{\xi}^{(i)}\right] u_{i}^{2}=0
$$

*) Di questo caso mi occupero in una prossima occasione. 
ed infine, soppresso il fattore $(\xi n)$ :

$$
\sum_{i}^{n+1} E_{\xi}^{(i)^{n}} E_{n}^{(i)^{n}} e_{\sigma}^{(i)} u_{i}^{2}=0 .
$$

Ne segue che $f_{\xi}$ ed $f_{\eta}$ sono polari reciproche precisamente rispetto a ciascuna delle $2^{n}$ quadriche-inviluppo:

$$
\sqrt{E_{\xi}^{\prime n} \bar{E}_{n}^{\prime n}} u_{1}^{2} \pm \sqrt{E_{\xi}^{\prime \prime n} \bar{E}_{n}^{\prime \prime n}} u_{2}^{2} \pm \cdots \pm \sqrt{E_{\xi}^{(n+1)^{n}} \bar{E}_{n}^{(n+1)^{n}}} u_{n+1}^{2}=0
$$

come del resto si puó facilmente verificare.

Pure al $\mathrm{n}^{\mathrm{o}}$ I4 si è osservato che se $f_{\xi}$ ed $f_{n}$ vengono a coincidere nella stessa $f_{\mu}$ di $[f]$ la $(34)$ si riduce alla:

$$
\beta_{\lambda} \beta_{\mu}^{n-1}=0 \text {. }
$$

Per stabilire quale inviluppo (40) corrisponda ad $f_{\sigma}$ nella polarita rispetto ad $f_{\mu}$, osservo che la (35) si puó porre sotto la forma:

$$
M_{\lambda}^{n+1}=\left(\xi_{n}\right) \alpha_{\lambda} \sum_{p=0}^{n} \alpha_{\xi}^{p} \alpha_{n}^{n-p}(\xi \lambda)^{n-p}(n \lambda)^{p} .
$$

Soppresso il fattore $\left(\xi_{\eta}\right)$, indi supposto che $f_{\varepsilon} f_{\eta}$ coincidano con $f_{\mu}, M_{\lambda}^{n+1}$ si riduce a:

$$
N_{\lambda}^{n+1}=\alpha_{\lambda} \alpha_{\mu}^{n}(\mu . \lambda)^{n}
$$

onde l'inviluppo $N_{\sigma}(N \beta)^{n}=$ o corrispondente ad $f_{\sigma}$ è quello di equazione:

$$
\alpha_{\sigma} \alpha_{\mu}^{n} \beta_{\mu}^{n}-n(\mu \sigma) x_{\mu}^{n}(\alpha \beta) \beta_{\mu}^{n-1}=0 .
$$

Si puó ora domandare quando (43) coincida coll'inviluppo $\beta_{\sigma} \beta_{\mu}^{n-1}=0$.

Risulta facilmente che cio avviene quando, e solo quando, sia

$$
(\mu \sigma) \alpha_{\sigma} \alpha_{\mu}^{n}=o \text {, }
$$

cioè quando $f_{\sigma}$ coincide con $f_{\mu}$, oppure colla quadrica di $[f]$ coniugata come luogo ad $f_{\mu}$. E cosi risolta una questione posta al gid citato $n^{\circ}$ I 4 .

Se ne deduce inoltre l'interessante proposizione che segue:

Se di due quadriche distinte la prima è coniugata come luogo rispetto alla seconda, l'inviluppo degli iperpiani secanti la prima in una quadrica coniugata come luogo alla sezione della seconda è polare della prima quadrica rispetto alla seconda; e reciprocamente.

\section{I fasci ciclici.}

22. Suppongo:

$$
j_{\lambda}^{3(n-3)}=\left(\alpha^{\prime} \alpha^{\prime \prime}\right)^{2}\left(\alpha^{\prime \prime} \alpha\right)^{2}\left(\alpha \alpha^{\prime}\right)^{2} \alpha_{\lambda}^{n-3} \alpha_{\lambda}^{\prime n-3} \alpha_{\lambda}^{\prime \prime n-3} \equiv 0,
$$

escludendo peró il caso in cui $\alpha_{2}^{n+1}$ sia potenza di una forma lineare. Con una scelta opportuna degli elementi di riferimento nel campo binario è allora lecito porre ${ }^{*}$ ):

$$
\alpha_{\lambda}^{n+1}=\lambda_{1}^{n+1}-\lambda_{2}^{n+1}
$$

*) Cfr. Marsano, La sestica binaria [Mem. Acc. Lincei, serie III, vol. XIX], Cap. I, Teor. VI. Ivi è citato il precedente lavoro dello stesso autore: Sopra due classi di forme binarie [Atti Acc. Lincei, I 883]. 
anzi, fissando convenientemente il punto unità in $S_{n}$ (e prendendo, come nel $§$ precedente, come fondamentale la piramide polare rispetto a tutte le quadriche di $[f]$ ), si può scrivere:

$$
e_{\lambda}^{(i)}=\lambda_{\mathrm{I}}-\eta^{i} \lambda_{2}
$$

dove con $n$ indico una radice $(n+1)$-esima primitiva dell'unità.

Il gruppo dei coni è quindi trasformato in sè dall'omografia binaria

$$
\lambda_{1} \lambda_{2}^{\prime}-n \lambda_{2} \lambda_{1}^{\prime}=0
$$

ciclica d'indice $n+\mathrm{I}$ e da ciascuna delle analoghe in corrispondenza alle rimanenti radici $(n+\mathrm{I})$-esime primitive dell'unitd.

Percio dico fascio ciclico il fascio in questione.

Posto:

(3)

risulta facilmente (per $p>0$ ):

$$
\Delta=\Delta_{\lambda}^{2}=\lambda_{1} \lambda_{2}
$$

$$
\left.\left(\alpha \alpha^{\prime}\right)^{2 p} \alpha_{\lambda}^{n-2 p+1} \alpha_{\lambda}^{\prime n-2 p+1}=-2\right\lrcorner^{n-2 p+1} .
$$

Le due quadriche del gruppo $\Delta_{2}^{2}=\mathrm{o}$ hanno dunque un posto notevole nella geometria del fascio e verranno dette le sue quadriche principali. Esse costituiscono gli elementi uniti di (2) e, come si deduce dalle (r), sono rispettivamente di equazione :

$$
\left\{\begin{array}{l}
r_{x}^{2} \equiv x_{1}^{2}+x_{2}^{2}+\cdots+x_{n}^{2}+x_{n+\mathrm{r}}^{2}=0, \\
s_{x}^{2} \equiv n x_{\mathrm{r}}^{2}+n^{2} x_{2}^{2}+\cdots+n^{n} x_{n}^{2}+x_{n+\mathrm{r}}^{2}=0 .
\end{array}\right.
$$

Dalla (2) del $\mathrm{n}^{0} 7$ e dalla (3) del presente $\mathrm{n}^{0}$ segue:

$$
\left(r^{\prime} r^{\prime \prime} \ldots r^{(k)} s^{\prime} s^{\prime \prime} \ldots s^{(n-k+\mathrm{I})}\right)^{2}=\alpha_{\mathrm{r}}^{k} \alpha_{2}^{n-k+\mathrm{I}},
$$

onde per $o<k<n+\mathrm{I}$ :

$$
\left(r^{\prime} r^{\prime \prime} \ldots r^{(k)} s^{\prime} s^{\prime \prime} \ldots s^{(n-k+1)}\right)^{2}=\mathrm{o} .
$$

Ciò : Per le due quadriche principali di un fascio ciclico si annullano gli $n$ invarianti $[\mathrm{I}, n] ;[2, n-\mathrm{I}] ; \ldots ;[n-\mathrm{I}, 2] ;[n, \mathrm{I}]$.

Reciprocamente, se per due quadriche distinte di $S_{n}$ si annullano gli invarianti $[\mathrm{I}, n] ;[2, n-\mathrm{I}] ; \ldots ;[n-\mathrm{I}, 2] ;[n, \mathrm{I}]$, senza che si annulli alcuno dei discriminanti $[0, n+\mathrm{I}] ;[n+\mathrm{I}, \mathrm{o}]$, le due quadriche sono quadriche principali di un fascio ciclico ${ }^{*}$ ). Cio si dimostra facilmente assumendo le due quadriche come fondamentali nella determinazione parametrica del campo binario.

Considero l'involuzione $I$ che ha per elementi doppi le quadriche principali, e cioè:

$$
\lambda_{1} \lambda_{2}^{\prime}+\lambda_{2} \lambda_{1}^{\prime}=0
$$

Se una quadrica $f_{\lambda}$ è $k$-armonica alla sua coniugata in $I$ si ha:

$$
\alpha_{\lambda}^{k} \alpha_{\lambda^{\prime}}^{n-k+1} \equiv \lambda_{1}^{n+1}-(-\mathrm{I})^{n-k+1} \lambda_{2}^{n+1}=0 ;
$$

*) Se si annulla anche il discriminante di una delle due quadriche, questa é l'unica quadrica specializzata del fascio; se si annullano entrambi i discriminanti il fascio è uno generico di quadriche tutte specializzate. 
onde per $n-k+$ I pari le quadriche soddisfacenti alla detta proprieta costituiscono il gruppo dei coni, mentre per $n-k+$ I dispari esse costituiscono il gruppo:

$$
\lambda_{1}^{n+1}+\lambda_{2}^{n+1}=0
$$

pure mutato in sè dall'omografia (2).

D'ora innanzi indico con $\Gamma_{i}$ il cono di $[f]$ corrispondente ad $e_{2}^{(i)}$. Osservo inoltre che, se $\chi$ è radice $(n+\mathrm{I})$-esima di - I ed a un tempo radice $2(n+1)$-esima primitiva di I, le quadriche del gruppo (7) corrispondono ai fattori lineari

$$
b_{\lambda}^{(i)}=\lambda_{1}-\chi^{2 i-1} \lambda_{2} \quad(i=1,2, \ldots, n+1),
$$

ed indico con $K_{i}$ la quadrica corrispondente ad $b_{\lambda}^{(i)}$.

A complemento dei precedenti risultati, si ha cosi:

Se $n$ è dispari:

r) La I trasforma in sè il gruppo dei coni, essendo in essa coniugati

$$
\Gamma_{i} e \Gamma_{i+\frac{n+1}{2}} \quad\left(i=1,2, \ldots, \frac{n+1}{2}\right) ;
$$

per $i$ due coni $\Gamma_{i}, \Gamma_{i+\frac{n+1}{2}}$ si annulla ciascuno degli $\frac{n+3}{2}$ invarianti

$$
[2 p, n-2 p+\mathrm{I}] \quad\left(p=0, \mathrm{I}, \ldots, \frac{n+\mathrm{I}}{2}\right) .
$$

2) La I trasforma in sè il gruppo (7), essendo in essa coniugate

$$
K_{i} \quad e \quad K_{i+\frac{n+1}{2}} \quad\left(i=\mathrm{I}, 2, \ldots, \frac{n+\mathrm{I}}{2}\right)
$$

per le due quadricbe $K_{i}, K_{i+\frac{n+1}{2}}$ si annulla ciascuno degli $\frac{n+\mathrm{I}}{2}$ invarianti

$$
[2 p+\mathrm{I}, n-2 p] \quad\left(p=0, \mathrm{I}, \ldots, \frac{n-\mathrm{I}}{2}\right) .
$$

Se $n$ è pari e si pone, come è lecito, $\chi=-n$, la I trasforma il gruppo dei coni nel gruppo (7), essendo in essa coniugati $\Gamma_{i}$ e $K_{l}$, dove è $l=\frac{i+1}{2}$ oppure $l=\frac{n+i+2}{2}$, secondo che $i$ è dispari o pari. Per $\mathrm{r}_{i}$ e $K_{l}$ si annulla ciascuno degli $\frac{n+2}{2}$ invarianti

$$
[2 p+\mathrm{I}, n-2 p] \quad\left(p=0, \mathrm{I}, \ldots, \frac{n}{2}\right) .
$$

L'enunciato I) è in un certo senso generalizzato come segue.

Le quadriche di $[f] k$-armoniche a $\Gamma_{i}$ vengono fornite dalla

(8)

ossia dalla

ed infine dalle

(9)

$$
\begin{gathered}
\eta^{(n-k+\mathrm{I}) i} \lambda_{\mathrm{I}}^{k}-\lambda_{2}^{k}=0, \\
\lambda_{1}^{k}-\left(\eta^{i} \lambda_{2}\right)^{k}=0, \\
\lambda_{1}-\psi^{i} \eta^{i} \lambda_{2}=0
\end{gathered}
$$$$
(t=\mathrm{o}, \mathrm{I}, \ldots, k-\mathrm{I}),
$$

dove $\psi$ è una radice $k$-esima primitiva dell'unita. Il gruppo (8), come si deduce dalle (9), è dunque costituito dalle quadriche trasformate di $\Gamma_{i}$ nelle successive potenze dell'omografia

$$
\lambda_{1}^{\prime} \lambda_{2}-\psi \lambda_{2}^{\prime} \lambda_{1}=0
$$

Rend. Circ. Matem. Palermo, t. XXIII (1907). - Stampato il s aprile I907. 
ciclica d'indice $k$. Il caso però che presenta maggior interesse è quello in cui $k$ è divisore di $n+\mathrm{I}$ e precisamente è:

E allora lecito porre:

$$
n+\mathrm{r}=k q \text {. }
$$

e le (9) divengono:

$$
\psi=n^{q}
$$

$$
\lambda_{1}-n^{q t+i} \lambda_{2}=0
$$$$
(t=0, \mathrm{I}, \ldots, k-\mathrm{I})
$$

Segue: Se $k$ è divisore di $n+\mathrm{I}$ ed $\dot{e} n+\mathrm{I}=k q$, $i$ coni di [f] si distribuiscono in $q$ gruppi di $k$ coni ciascuno, appartenendo ad uno stesso gruppo due coni $K_{i}, K_{l}$ pei quali sia $i \equiv l$ mod $q$. Per due coni di uno stesso gruppo si annulla ciascuno dei $(q+\mathrm{I})$ invarianti $[k p, k(q-p)](p=0, \mathrm{I}, \ldots q)$.

23. Termino con un breve cenno sulla schiera associata $\left(\mathrm{n}^{\mathrm{i}} \mathrm{I} 3,20\right)$ per il caso del fascio ciclico. Se si osserva che il primo gruppo polare di una delle quadriche principali rispetto al gruppo dei coni è costituito dall'altra contata $n$ volte, risulta facilmente che la schiera associata ad [f] è quella individuata dalle due quadriche principali considerate come inviluppo.

Se ne deduce che $[f]$ e la schiera sono polari reciproci rispetto a ciascuna delle $2^{n}$ quadriche rispetto alle quali sono polari reciproche le due quadriche principali. Quindi:

I) Sussiste il teorema di reciprocità (qualunque sia $n$ ).

2) La schiera associata ad un fascio ciclico è ciclica ed ba per inviluppi principali le quadriche principali del fascio, pensate come inviluppo.

Per il caso di $n$ pari, che si ridurrebbe ad una particolarizzazione di risultati precedenti, mi limito ad osservare che l'involuzione di cui si tratta nell'enunciato in fine del $\mathbf{n}^{\circ} \mathbf{1}$, coincide con $I$.

Per $n$ dispari, posto come altrove

osservo che si ha

$$
\varepsilon_{\lambda}^{(i)}=\alpha_{2}\left(\alpha E^{(i)}\right)^{n}
$$

ed, essendo

$$
\varepsilon_{\lambda}^{(i)}=E_{2}^{(i)^{n}} \lambda_{1}+E_{1}^{(i)^{n}} \lambda_{2},
$$

anche

$$
E_{1}^{(i)^{n}}=\mathrm{I}, \quad E_{2}^{(i)^{n}}=n^{-i},
$$

Se suppongo

$$
\varepsilon_{\lambda}^{(i)}=n^{-i} \lambda_{1}+\lambda_{2} \text {. }
$$

$$
l-i \equiv \frac{n+\mathrm{I}}{2}, \bmod (n+\mathrm{I}) \quad(0<l \leqslant n+\mathrm{I})
$$

onde

segue

ossia

$$
n^{l-i}=-\mathrm{I},
$$

$$
\varepsilon_{\lambda}^{(i)}=-n^{-l}\left(\lambda_{1}-n^{l} \lambda_{2}\right),
$$

$$
\varepsilon_{\lambda}^{(i)}=-\eta^{-l} e_{\lambda}^{(l)} \text {. }
$$

Dunque: Se $\Gamma_{i}, \Gamma_{1}$ sono due coni coniugati in $I$, nellap rojettività posta al $n^{\circ}$ I 3 fra [f] e la schiera associata, a $\Gamma_{i}$ (risp. a $\Gamma_{l}$ ) corrisponde l'inviluppo specializzato avente 
per nucleo una quadrica giacente nella faccia della piramide fondamentale opposta al vertice di $\Gamma_{l}$ (risp. di $\Gamma_{i}$ ).

La projettivita del $\mathrm{n}^{\circ}$ I 3 risulta dunque dal prodotto dell'involuzione $I$ in $[f]$, per la projettività, tra $[f]$ e la schiera, subordinata alle polarità che scambiano l'una nell'altra le quadriche principali.

Osservo infine che le ultime considerazioni si possono mettere in rapporto con quelle del $\mathrm{n}^{\circ} 2 \mathrm{I}$, assumendo come gruppo $M_{\lambda}^{n+\mathrm{r}}=0$ il gruppo (7) del $\mathrm{n}^{\mathrm{o}} 22$.

Sondrio, 2 I gennaio 1907.

LUIGI BRUSOTTI. 